Bending continuous structures with SMAs: a novel robotic fish design

This article has been downloaded from IOPscience. Please scroll down to see the full text article.

2011 Bioinspir. Biomim. 6045005

(http://iopscience.iop.org/1748-3190/6/4/045005)

View the table of contents for this issue, or go to the journal homepage for more

Download details:

IP Address: 138.100.76.136

The article was downloaded on 30/11/2011 at 10:40

Please note that terms and conditions apply. 


\title{
Bending continuous structures with SMAs: a novel robotic fish design
}

\author{
C Rossi, J Colorado, W Coral and A Barrientos \\ Centre for Automation and Robotics, Universidad Politécnica de Madrid, Madrid, Spain \\ E-mail: claudio.rossi@upm.es
}

Received 30 April 2011

Accepted for publication 20 September 2011

Published 29 November 2011

Online at stacks.iop.org/BB/6/045005

\begin{abstract}
In this paper, we describe our research on bio-inspired locomotion systems using deformable structures and smart materials, concretely shape memory alloys (SMAs). These types of materials allow us to explore the possibility of building motor-less and gear-less robots. A swimming underwater fish-like robot has been developed whose movements are generated using SMAs. These actuators are suitable for bending the continuous backbone of the fish, which in turn causes a change in the curvature of the body. This type of structural arrangement is inspired by fish red muscles, which are mainly recruited during steady swimming for the bending of a flexible but nearly incompressible structure such as the fishbone. This paper reviews the design process of these bio-inspired structures, from the motivations and physiological inspiration to the mechatronics design, control and simulations, leading to actual experimental trials and results. The focus of this work is to present the mechanisms by which standard swimming patterns can be reproduced with the proposed design. Moreover, the performance of the SMA-based actuators' control in terms of actuation speed and position accuracy is also addressed.
\end{abstract}

(Some figures in this article are in colour only in the electronic version)

\section{Introduction}

Underwater creatures are capable of high performance movements in water. Thus, underwater robot design based on the mechanism of fish locomotion appears to be a promising approach. Over the past few years, researchers have been developing underwater robots inspired by the swimming mechanism of fish [1-6]. Yet, most of them still rely on servomotor technology and a structure made of a discrete number of linear elements, exceptions being the Airacuda by FESTO, which adopts pneumatic actuators, and the MIT fish [7], that has a continuous soft body, and a single motor produces a wave that is propagated backward in order to generate propulsion.

In fact, actuation technology in robotics is dominated by two kind of actuators: electric motors/servomotors and pneumatic/hydraulic. In mobile robotics, the former is mostly used, with exceptions being e.g. large-legged robots. The (rotatory) motion of the motors is transmitted to the effectors through gearboxes, bearings, belts and other mechanical devices where linear actuation is needed. Although applied with success in uncountable robotic devices, such systems can be complex, heavy and bulky ${ }^{1}$. In underwater robots, propellers are mostly used for locomotion and maneuvering. However, propellers may have problems of cavitation, noise, efficiency, can get tangled with vegetation and other objects, and can be dangerous for sea life.

Alternative actuation technology in active or 'smart' materials has opened new horizons as far as simplicity, weight and dimensions. Materials such as piezo-electric fiber composites, electro-active polymers and shape memory alloys (SMAs) are being investigated as promising alternatives to standard servomotor technology. The potential gain in weight and dimension would allow for building lighter, simpler and smaller robots.

In order to reproduce the undulatory body motion of fishes, smart materials appear to be extremely suited. In fact,

\footnotetext{
1 Robotuna, a robot fish developed at MIT in 1994, has 2843 parts controlled by six motors (source: MIT News, http://web.mit.edu/newsoffice/2009/robo-fish-0824.html).
} 
over the last few years, there has been increasing activity in this area. Within the field of underwater locomotion, research about the use of smart materials is mainly focused on mechatronics design and actuation control. As far as mechatronic design is concerned, much work is devoted to building hydrofoils using, e.g., piezo-electric fiber composite $[8,9]$ or embedding SMA wires into an elastic material such as silicone [10-13]. SMAs are also used as linear actuators in articulated structures [14-16]. Finally, emerging materials such as electro-active polymers are adopted in [17-20].

In this work, we have adopted SMAs as actuation technology mainly due to their advantage of working at low currents and voltages. SMAs are also extremely cheap and easily available commercially. Due to the relative novelty of smart material technology, the literature and the know-how regarding their use is not consolidated yet. The accurate control of such materials still remains an important challenge to tackle. In terms of control, excellent results have been achieved in [21, 22], demonstrating that using the proper control, NiTi SMA wires can be surprisingly fast in contrast with previous approaches found in the literature, and the general belief that their response speed is limited because of slow heat transfer characteristics [23, 24] and the long transient associated with the phase transformation process $[25,26]$.

In this paper, we present our work on a swimming biomimetic fish robot. The main characteristics of this robot are (i) the concept of a continuous bendable structure of the robot actuated by a discrete number of (smart) actuators, and (ii) the complete lack of any standard mechanical parts and rotating actuators, i.e. a gear-less and motor-less robot. These are distinctive features of our prototype. In fact, the prototypes cited above either use SMAs to actuate classical articulated (rigid) bodies, or are used for bending hydrofoils for imitating oscillating fins.

The paper is organized as follows. Section 2 introduces the novel mechatronic design. Section 3 presents the SMA modeling and the proposed control methodology, that ensures a feasible use of the SMA-based actuators as far as precision and actuation speed. Section 4 briefly describes swim patterns in fish, highlighting the key aspects for mimicking fish locomotion using the artificial counterpart. Section 5 presents simulations related to the motion patterns, with the aim of demonstrating the feasibility of the concept. Also it introduces the performance metrics for assessing the performance of the concept both in simulation and experimentally. Section 6 reports the experimental results obtained with the prototype in water. Finally, section 7 concludes the paper with closing remarks.

\section{Mechatronics concept design}

\subsection{Biological foundations}

Fish swim bending their body, producing a backwardpropagating propulsive wave. Such bending comes in different ways (see figure 1). Anguilliform swimmers show a snakelike motion: their body can be divided into numerous segments from head to tail to reproduce at least one complete wavelength
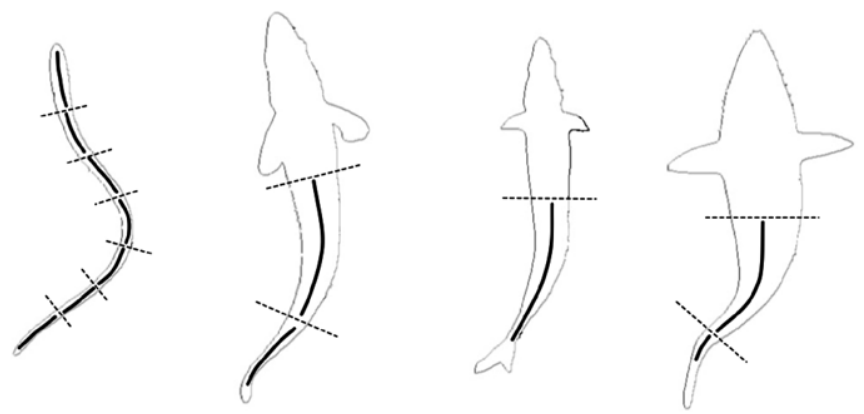

Figure 1. Different kinds of undulatory swimming: from left to right: anguilliform, subcarangiform, carangiform, thunniform (figure adapted from [27]).

along the body. Conversely, subcarangiform, carangiform and thunniform swimmers only bend roughly the second half of the body, and the number of segments is reduced to 1 or 2 [27].

Ellerby et al [28] explain that during steady swimming most fish power swim with their lateral musculature. Muscular contraction, and the mechanical properties of the passive components of the body, combine to produce a wave of curvature that passes along the fish from head to tail, in which lateral muscle fibers lengthen and shorten rhythmically. At low-tailbeat-frequency during sustained swimming, slowtwitch aerobic muscles power the motion. Our mechatronic concept takes inspiration from such arrangement and imitates the red or slow-twitch muscles (see inset in figure 2), that fishes use during steady swimming, by means of SMA-based muscles.

In fish, muscle fibers are used for bending a flexible but nearly incompressible axis. Such an axis is either composed of a (visco)elastic beam (notochord) or a series of vertebrae connected through intervertebral discs. The main structure of our robot fish is inspired by this principle and is composed of a continuous flexible backbone.

\subsection{The concept}

For our model, we have chosen to divide the body in three segments of the same length (cf figure 2). The reduced number of equally sized segments simplifies the study and the implementation of swimming patterns, while having enough degrees of freedom that allow for different swimming modes to be reproduced. Such subdivision allows reproducing subcarangiform, carangiform and thunniform swimming modes (figure 3), and even anguilliform swimming, although for the last mode a higher number of segments is usually employed.

A key concept of our prototype is using SMA actuators to bend a continuous flexible structure, representing the backbone of the robot fish, made of polycarbonate of $1 \mathrm{~mm}$ thickness. This material has been chosen for its temperature, impact resistance and flexibility. An additional structure of ribs is employed to support the silicon-based skin that provides the three-dimensional shape of the robot (figure 4). The overall length of the fish is $30 \mathrm{~cm}$ (not including the caudal fin). 


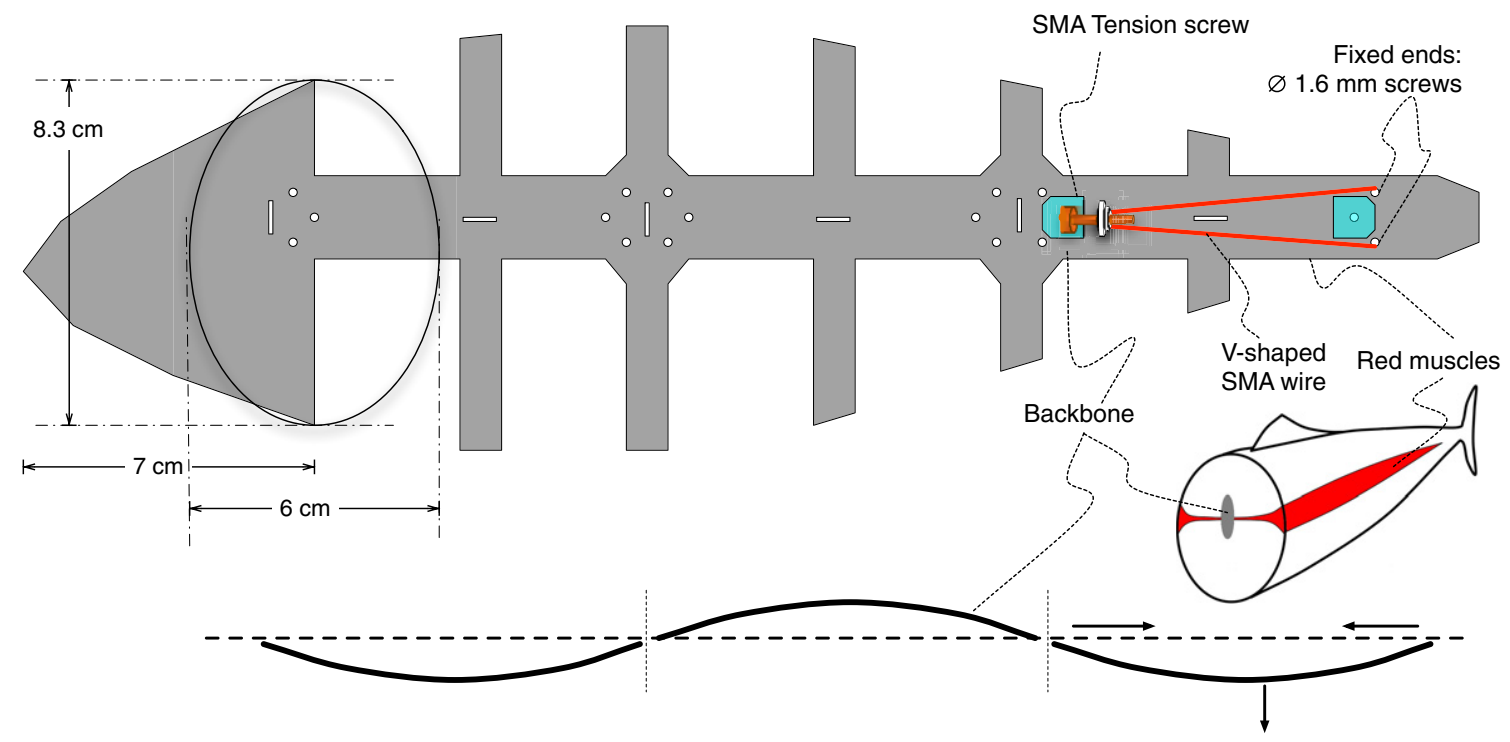

Figure 2. Lateral and upper views of the deformable structure (see also figures 4 and 5). The inset shows the location of the red muscles in real fish.

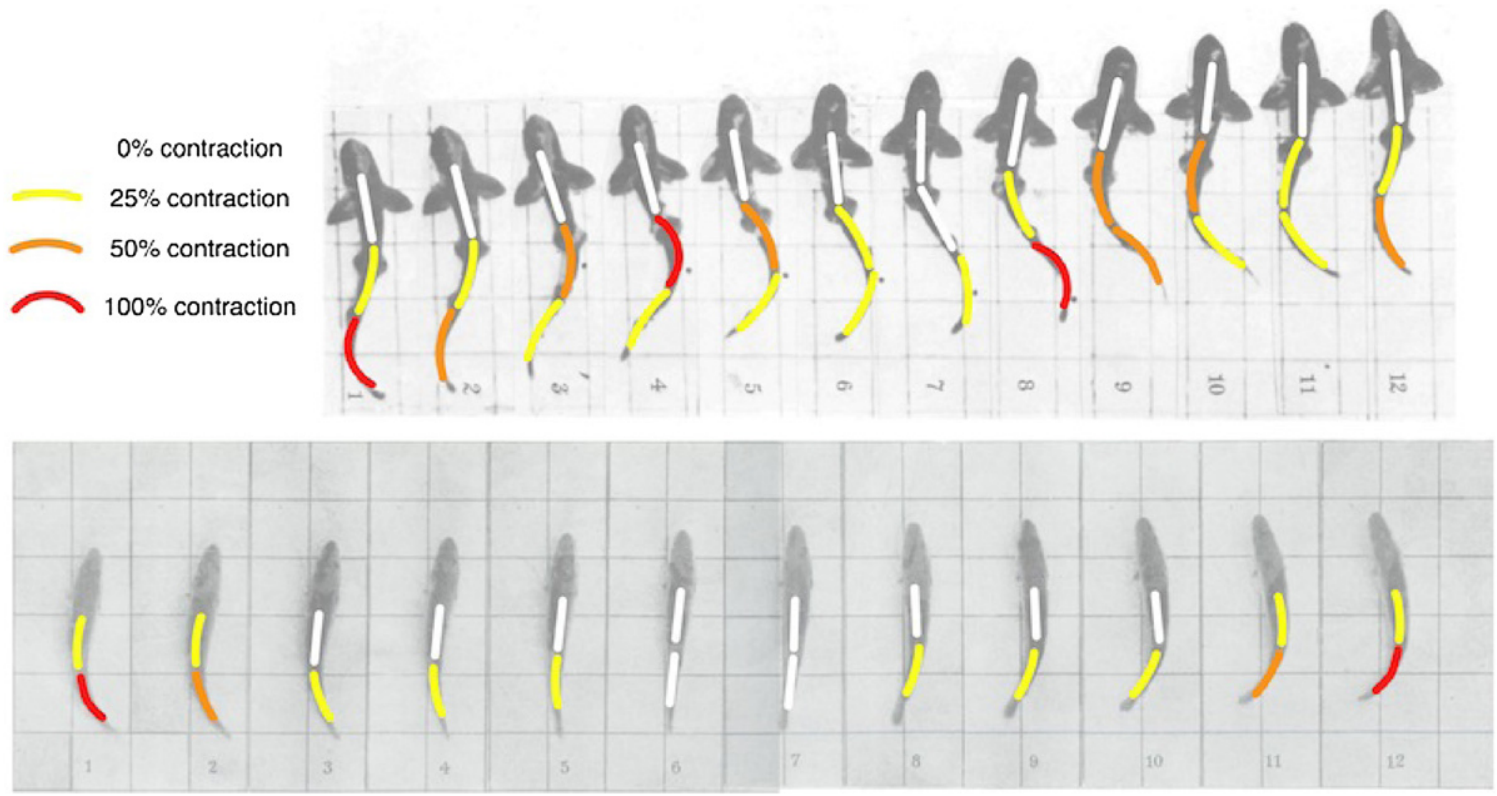

Figure 3. Stills of the undulatory movement of body and tail segments (adapted from [29]). The proposed fish model can imitate both subcarangiform (top) and carangiform (bottom) swimming. The percentage of contraction refers to the SMA contraction that bends the corresponding body segment.

Six SMA-based actuators are employed. Their length is $1 / 3$ of the body length (i.e. $8.5 \mathrm{~cm}$, not counting the caudal fin and the head) and are positioned in pairs, parallel to the body in such a way to produce antagonistic movement. This antagonistic configuration of SMA wires has some advantages in terms of increasing the range of controllable actuation, since both directions of motion (contraction and elongation) can be actively controlled. Figure 2 shows the location of the SMA wires within the skeleton structure of the prototype.

A novel $V$-shaped configuration of the wires, where each artificial muscle is composed of a single V-shaped SMA wire, twisted around the tension screw, is shown in figure 2. It allows us to double the pull force without a significant increase of power consumption.

Under nominal operation of the SMAs, our muscle-like SMA arrangement can bend the body segments up to $28^{\circ}$ (angle $\beta$ of figure 5, see also figure 7), regardless of the fact that SMA wires only contract approximately $4 \%$ of their length. By increasing the input electrical current and including a suitable control that handles an overloaded SMA operation, contraction time of $0.5 \mathrm{~s}$ can be achieved, and strain can be increased up to $6 \%$, corresponding to a bending of approximately $36^{\circ}$. Note that the passive SMA does not need to stretch. This is explained by the mechanical arrangement, since the radius of 


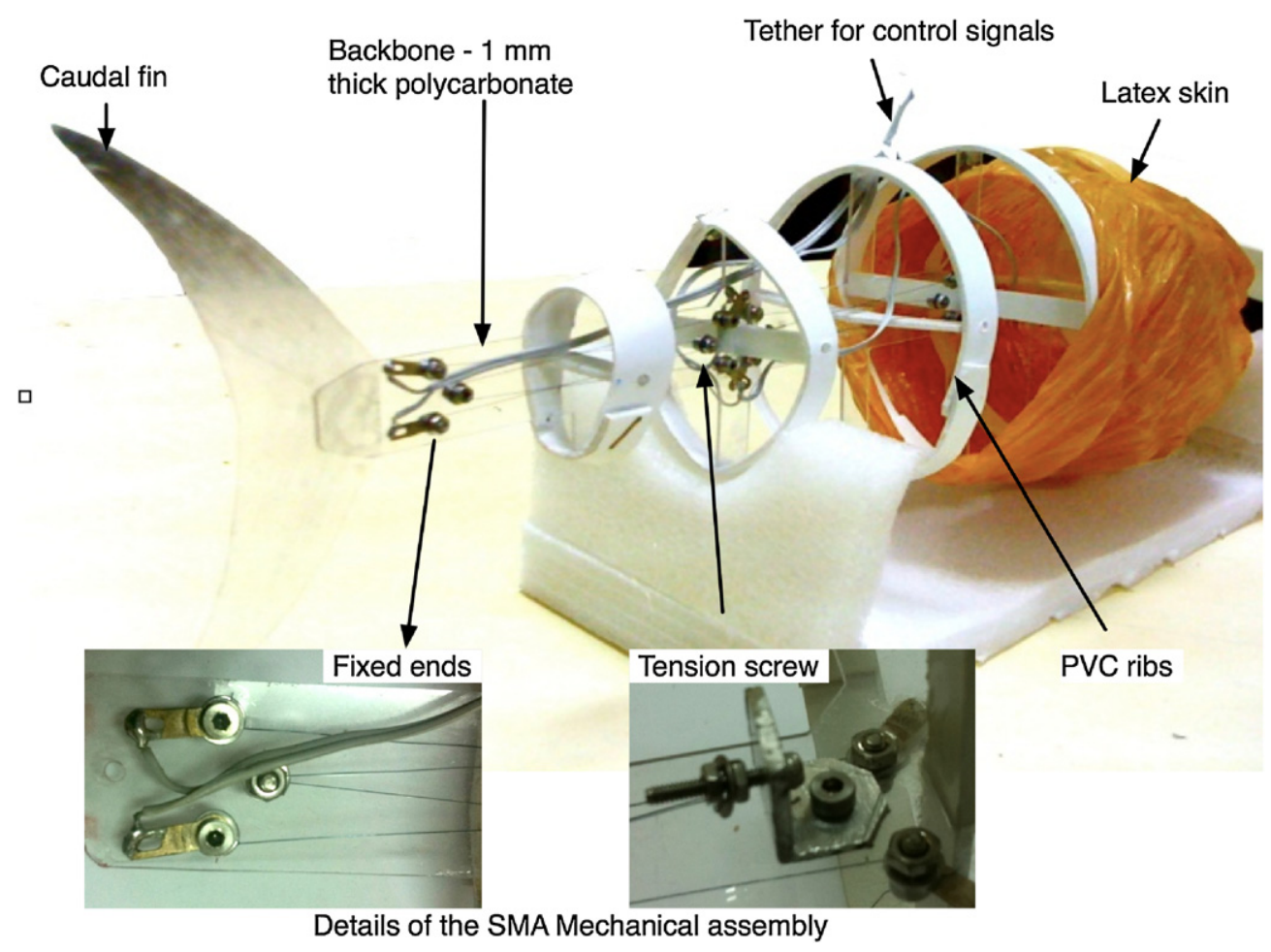

Figure 4. Fish skeleton structure including a latex-based skin for water protection.

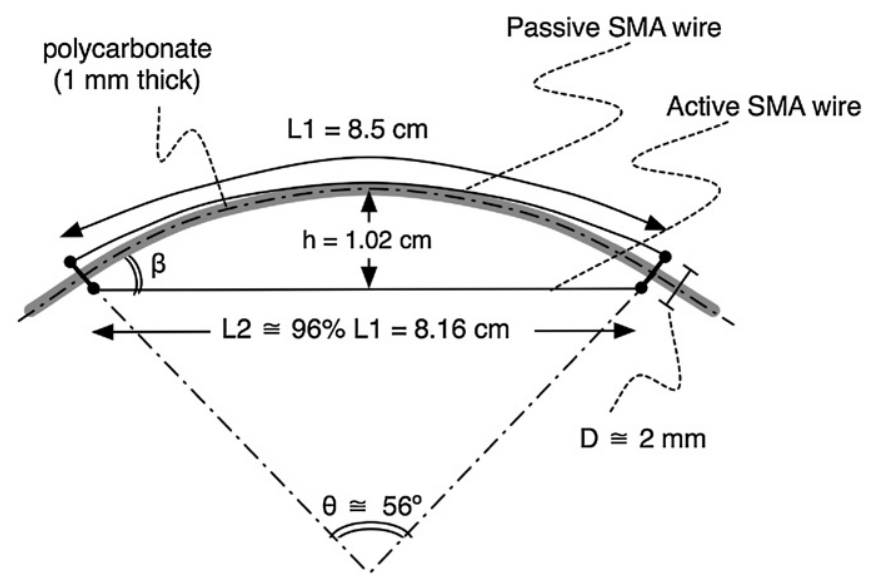

Figure 5. Principle of the bendable polycarbonate structure. The V-shaped SMA wires are parallel to the spine segment. As one contracts, it causes the polycarbonate strip to bend. L1 is the SMA length at rest, and L2 is the contracted SMA length. The

relationship is $L_{2} / L_{1}=$ chord/arc $=\sin (\theta / 2) /(\theta / 2)$, where $\theta$ is the central angle of the circle sector and $h$ is the arc height. For nominal SMA operation, contraction in the wires causes $\theta \approx 56^{\circ}$, and $\beta=\theta / 2 \approx 28^{\circ}$.

the arc that forms the passive SMA is larger than the radius of the arc that forms the polycarbonate strips.

\subsection{Shape memory alloy-based actuators}

SMAs are materials capable of changing their crystallographic structure (from austenite and martensite phases) due to changes in their temperature. In SMA wires, such temperature changes can be produced by an electrical current. Joule heating causes a phase change, and the SMA returns to its memorized shape by contracting. In this way, the SMA wire can be used as a linear actuator.

The SMA used, Nitinol, one of the most commercially available SMAs, is an alloy of nickel and titanium (NiTi). It is characterized by a high recovery stress $(>500 \mathrm{MPa})$, low operational voltage (4-5 V), a reasonable nominal operational strain $(\approx 4 \%)$ and a long life (up to $10^{6}$ cycles). We have adopted a SMA NiTi wire with a diameter size of $150 \mu \mathrm{m}$ that has a pull force of $230 \mathrm{~g}$ at consumption of $250 \mathrm{~mA}$ at room temperature, and a nominal contraction time of $1 \mathrm{~s}$. The diameter size of the wires has been chosen as a trade-off between current consumption, pull force and contraction time.

In terms of modeling and control, the behavior of SMAs is more complex than many common materials: the stressstrain relationship is nonlinear, hysteresis is present, large reversible strains are exhibited and it is temperature dependent. For these reasons, a low-level control electronics has been developed for a closed-loop position control precise enough for the application at hand. Control electronics is described in section 3.3.

\section{SMA modeling and control}

Control design and tuning is highly dependent on a modeling stage. In terms of SMA modeling, there have been numerous models proposed to capture or explain the characteristics of SMAs, most notably in terms of their thermo-mechanical relations and the hysteresis effects [30]. Most of these models are also known as phenomenological models. Some of them were used for control purposes, including the Kuribayashi model based on experimentally identified thermo-mechanical 


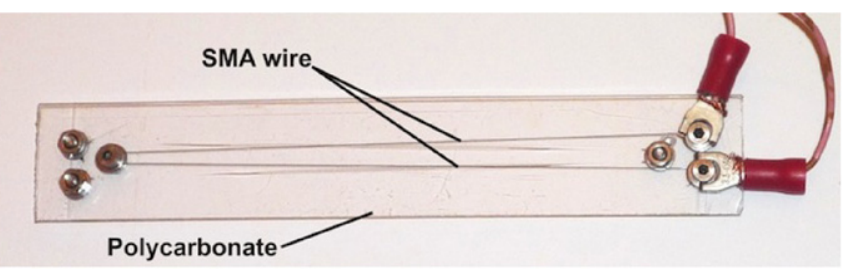

Figure 6. The test mock-up uses the V-shape configuration. The mock-up corresponds to a segment of the fish's backbone, which is a $10 \times 2 \mathrm{~cm}^{2}$ stripe of $1 \mathrm{~mm}$ thick polycarbonate, with a $174 \mathrm{~mm}$ long SMA wire in a V-shaped configuration.

relations [31], the sublayer models of Ikuta et al [32] and Tanaka's constitutive model [33].

Despite phenomenological models providing a good insight into SMA behavior, herein we attempt to simplify the modeling stage by finding an accurate linear approximation that matches the behavior of our SMA actuator.

\subsection{SMA identification}

SMA actuators have generally been considered to have slow response due to a large transition from heating to cooling, and also due to the inherent thermal hysteresis. The common method in actuation is by electrical heating. The large hysteresis loop, as well as the nonlinear characteristic of the phase transformations, also makes SMA actuators difficult to control accurately.

Nonetheless, by using an antagonistic configuration of a pair of SMA wires, experiments conducted in [21, 34-36] have demonstrated some advantages in terms of controllability. When the active actuator is being heated while the passive (antagonistic) is cooling, hysteresis effects are reduced due to the external stress that the active actuator applies on the inactive one above the austenite finish temperature. On the other hand, the change of SMA electrical resistance depends on martensite ratio and strain. The former is a highly nonlinear and hysteretic function of temperature and stress, whereas the latter is linear.

Using the mock-up shown in figure 6, we have verified that the hysteresis on the electrical resistance curve was smaller than the hysteresis on the temperature curve. This issue suggests that a linear approximation relating the electrical resistance change $R(t)$ and the input current $I(t)$ would be sufficient to model how our SMA actuator actually responds to heating.

The system identification process performed on the mock-up determines how much input current $(I)$ must be applied to the SMA actuators in order to achieve a desired electrical resistance value $(R)$ that finally provides the desired bending angle $(\beta)$ of the fish's backbone structure. This resistance/angle relationship is also linear, as experimentally demonstrated in section 3.4, figure 12 .

Figure 7 shows the performance of the mock-up when applying an input current of $300 \mathrm{~mA}$. The angle shown in the figure is in good accordance with the theoretical value of $28^{\circ}$ shown in figure 5 . Note that the wires' speed and strain depends on how fast and by how much the wire temperature

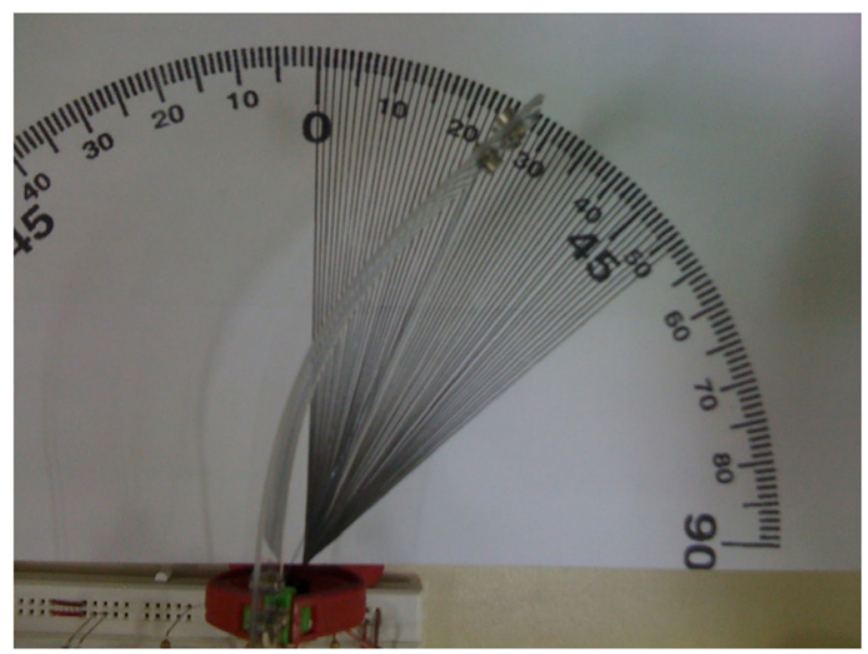

Figure 7. Experimental angle (bending the structure), $\beta \approx 28^{\circ}$ at $300 \mathrm{~mA}$ current.

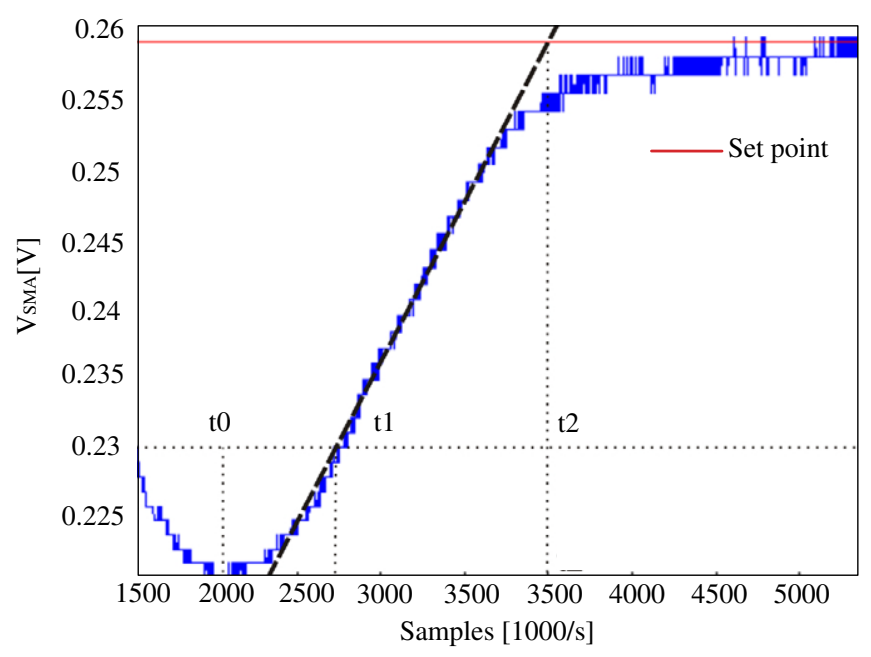

Figure 8. Voltage SMA versus a given set point. $y_{1}=0.258, y_{0}=$ $0.232, t_{1}=2.725, t_{0}=2.2, t_{2}=4, u_{1}=1, u_{0}=0$, experimentally determined.

is increased. In our tests, we have verified that both response time and percentage of contraction can be increased, without damaging the SMA, feeding it with a current of about $500 \mathrm{~mA}$ (see figure 11).

Therefore, the aforementioned identified linear model relating the electrical resistance change $R(t)$ and the input current $I(t)$ has been defined using the following first-order transfer function:

$$
\frac{R(s)}{U(s)}=\frac{K_{0}}{1+s \tau_{0}}=\frac{0.011}{0.52 s+1},
$$

where the coefficients $K_{0}=0.011$ and $\tau_{0}=0.525$ are obtained from the response of the open loop system to a step input $U(t), U(t)$ being the input current $I(s)$ delivered by the controller ${ }^{2}$.

The resistance output $R(t)$ is then mapped to voltage measurements $(V=I R)$. Figure 8 shows the response of

2 The step input used was software generated using NI LabVIEW and associated D/A control hardware. 


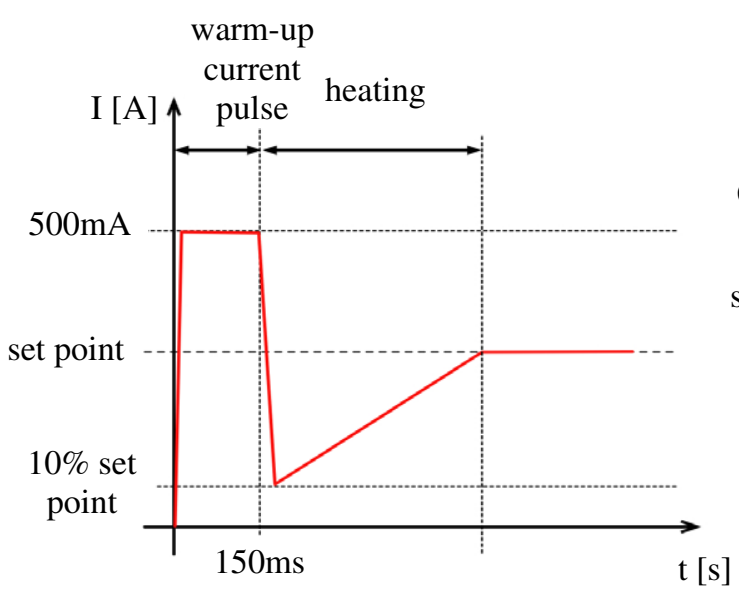

(a)

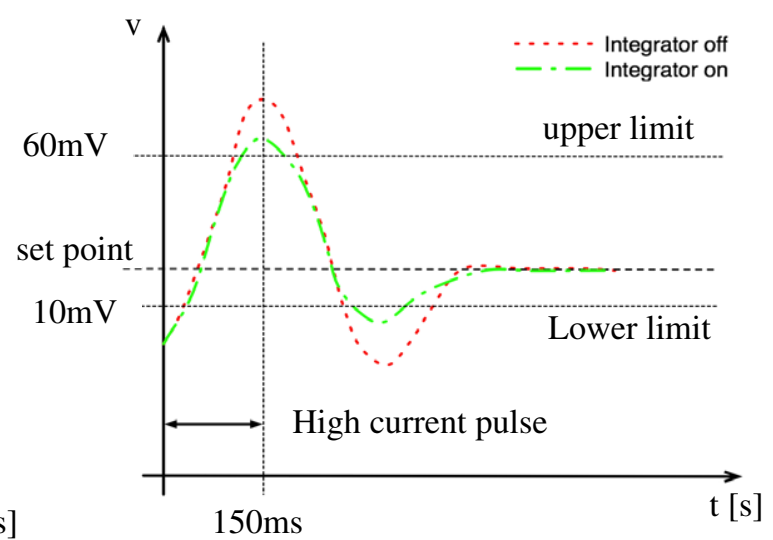

(b)

Figure 9. Control strategy: (a) pre-heating and heating mechanism, $(b)$ behavior of the control signal.

the model, registering the transition to stabilization stage at $y(t)=y_{0}$ to $U(t)=u_{0}$; a step input is applied from $u_{0}$ to $u_{1}$.

In order to validate the response of the model in (1), the appendix reports SMA simulation results obtained by using a phenomenological nonlinear model that confirms the accuracy of the lineal approximation adopted herein. Discussion comparing this model to experimental testing using the identified linear model is presented, while table A1 summarizes the thermo-mechanical equations of this phenomenological model. The results confirm that the model in (1) is suitable for developing a PID controller that regulates the change of electrical resistance based on the applied input current, furthermore servoing electrical resistance to feedback the bending angle of the fish robot's backbone. The next section introduces the control strategy and the mechanisms that ensure precise robot motion.

\subsection{Controller setup}

The accurate control of the bendable structure of the fish presents two main challenges due to the behavior of the SMAs when switching from heating to cooling phases: (i) slack in the fibers and (ii) limited actuation speed.

Slack issues occur when fixing the wires in an antagonistic configuration. The SMA's wires contract when heated, and extend upon cooling even if there is no tension applied on them. As a consequence, the passive wire can develop a few millimeters of slack as it cools. This behavior is known as two-way shape memory effect, as explained in [21].

On the other hand, the limitation in actuation speed occurs due to the large switching time between cooling and heating phases (about $0.5 \mathrm{~s}$ during nominal operation for the application at hand). It compromises the actuation speed of the antagonistic configuration.

To solve both issues, a pre-heating mechanism has been introduced within the closed-loop control architecture. This mechanism works by avoiding the temperature on both wires dropping below $10 \%$ of the maximum applied electrical current. This issue improves the speed of the SMA-based actuators due to the fact that an already-warm wire can begin to increase its pull immediately the heating current is raised, whereas a cold wire must first be raised to operating temperature.

Thus, by ensuring two conditions: (i) an already-warm wire, and (ii) inducing external stress (tension applied by the active actuator), the SMA wire of the inactive actuator can be stretched faster and slack issues are avoided. The pre-heating mechanism is shown in figure 9. Once the preheating mechanism is turned on, the minimum threshold of applied current is about $50 \mathrm{~mA}$ (10\% of the maximum current, as shown in figure $9(a))$. This prevents the inactive alloy from complete cooling. On the other hand, the active alloy must induce an external stress on the passive one in order to speed up the recovering shape memory process during the cooling phase. This external stress during the actuator's cooling is generated due to both the damping effect of the fishbone and the active SMA's pull force (see also figure $15(b)$ and $(d))$.

The pre-heating step is implemented by the control system, that sends a high pulse of current only for a small period of time, $500 \mathrm{~mA}$ during $150 \mathrm{~ms}$. Then, it feeds the SMA with a ramp from $10 \%$ of the target current to $100 \%$ of the target current.

For the control strategy, we have used a PID controller. Using the Ziegler-Nichols methodology, we have tuned the values of the PID parameters $\left(K_{p}, T_{i}, T_{d}\right)$ based on the analysis of the system under both opened/closed loops. The system has been represented by the identified model in (1).

From the experimental data corresponding to figure 8, we can compute the following parameters:

$\tau_{0}=t_{1}-t_{0}, \quad \gamma_{0}=t_{2}-t_{1}, \quad K_{0}=\left(y_{1}-y_{0}\right) /\left(u_{1}-u_{0}\right)$. According to Ziegler-Nichols, the relations among these coefficients and the controller parameters are

$$
K_{p}=\left(1.2 \gamma_{0}\right) /\left(K_{0} \tau_{0}\right), \quad T_{i}=2 T_{0}, \quad T_{d}=0.5 .
$$

Then, according to the $Z$ domain transformation, the discrete PID controller is

$$
U(z)=E(z) K_{p}\left[1+\frac{T}{T_{i}\left(1-z^{-1}\right)}+T_{d} \frac{1-z^{-1}}{T}\right] .
$$




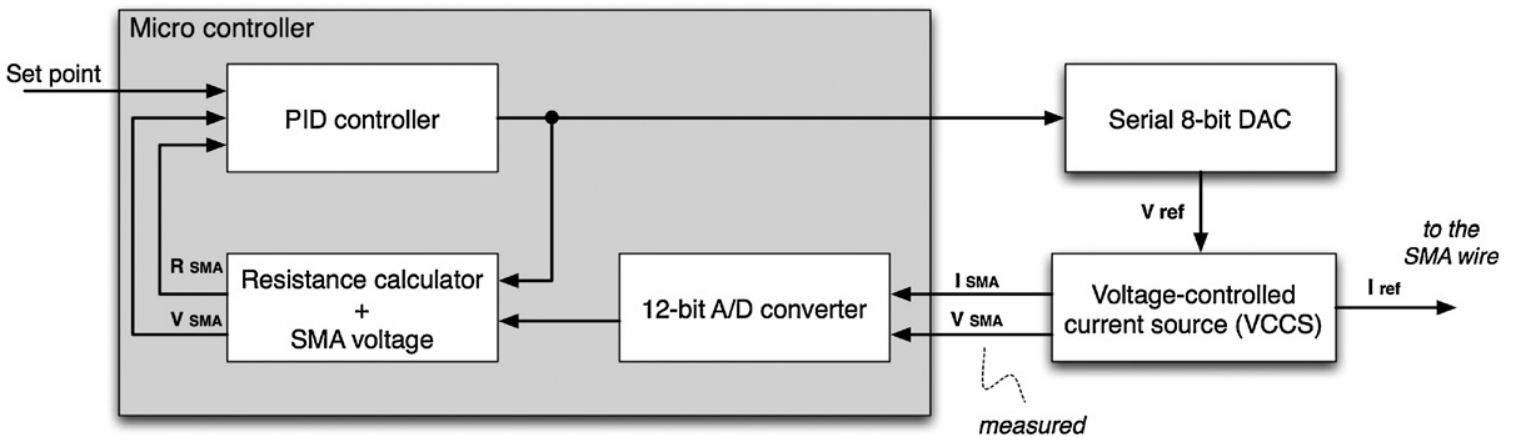

Figure 10. Closed-loop control of a single SMA actuator.

Also

$$
\frac{U(z)}{E(z)}=a+\frac{b}{1-z^{-1}}+c\left(1-z^{-1}\right)
$$

being

$$
a=K p, \quad b=\frac{K_{p} T}{T_{i}}, \quad c=\frac{K_{p} T_{d}}{T},
$$

where $E(z)$ is the measured position error of the actuator (bending angle $\beta$ ) and $U(z)$ is the SMA actuator driven current. To achieve $E(z)$, the system feeds back the electrical resistance change of the SMAs $(R)$, and calculates the bending angle based on this measurement. The next section details this resistance/position relationship and the control results.

The parameters of the PID controller are $K_{p}=$ 97.22, $T_{i}=1.05, T_{d}=0.2625$. The discrete controller parameters are calculated using $T=0.1<\tau_{0} / 4$. These parameters are also tuned for a stable SMA response in open loop, corresponding to the values $a=97.22, b=1.05, c=$ 255.202. This tuning process is based on the response of the SMAs using a step signal input of $260 \mathrm{mV}$ that corresponds to $300 \mathrm{~mA}$ of SMA arousal.

\subsection{Control architecture}

As pointed out earlier, SMAs provide the possibility of developing control systems without the need of external sensor hardware. The detection of inner electrical resistance in fact allows an indirect measurement of the temperature, and based on this, regulating the actuator's movement [34]. Based on this approach, the control electronics is composed by a micro controller implementing the PID algorithm described earlier. The PID controller receives the input reference position (set point) and the feedback of SMA's voltage and current; therefore, it calculates the heating current to drive the SMA actuator. The digital output of the PID controller is converted to a reference current in two steps. First, it is converted into an analog signal using a 2-wire serial 8 bit DAC (digital to analog converter) with rail-to-rail outputs. Then, a voltage controlled current source (VCCS) transforms the dc voltage into a constant current that feeds the SMA. This stage has been designed to have a low power consumption $(<10 \mathrm{~mA})$. Figure 10 shows the closed-loop control block diagram.

The DAC output goes from 0 to $5 \mathrm{~V}$ with a resolution of $0.02 \mathrm{~V}$. The frequency is set to $400 \mathrm{kHz}$ in order to avoid excessive current in the SMA. The measured voltage $\left(V_{\text {SMA }}\right)$ and current $\left(I_{\text {SMA }}\right)$ on the SMA fiber are fed back to the micro controller in order to close the loop using the PID controller (equation 3). Since the hardware used (16F690-PIC) has a 12 bit A/D converter, and the maximum voltage measured at the SMA is $V_{\mathrm{SMA}}=0.55 \mathrm{~V}$, we have obtained a resolution of $0.537 \mathrm{mV}$.

On the other hand, taking into consideration the maximum current through the wire (500 mA), we can measure SMA resistance variations about $1.074 \mathrm{~m} \Omega$. Therefore, since the maximum variation in the SMA length is $0.34 \mathrm{~cm}$, and the maximum variation of the resistance is $1.6 \Omega$, the theoretical position error of the system based on the SMA length is $0.067 \%$, i.e. $0.12 \mathrm{~mm}$.

\subsection{Control results}

Using the mock-up in figure 6 , we have tested the control system by applying different target resistances (i.e. desired bending angles $\beta$ ). In order to assess the precision of the system, we have measured the final resistance of the SMA wire.

Figure 11 shows the comparison of the transient period of electrical resistance curves as a function of five current profiles, ranging from 300 to $500 \mathrm{~mA}$. Figure 11 (left) shows the variation of the SMA resistance under open-loop behavior, whereas figure 11 (right) shows the closed-loop behavior of the system using the PID controller with the preheating mechanism. The setup reference corresponds to a resistance value of $12.25 \Omega$, which corresponds to a bending angle of $\beta=11^{\circ}$.

The linear relationship between the resistance change and the bending angle $\beta$ of the structure has been experimentally measured (cf figure 12). The micro-controller measures the resistance of the SMA and computes the relation $R=$ $-0.0245 \beta+12.5$ in order to feed back the angular position of the bending structure and regulate it using the PID controller (see figure 11 (right)).

Table 1 summarizes the results for different set-points (target resistance) and the resulting bending error. The length error has been calculated as function of the resistance error $^{3}$

3 The maximum contraction of the wire can be measured as $\Delta L_{A_{f}}=$ $\left(R_{M f}-R_{A f}\right) L_{R}$, where $R_{M f}(\Omega)$ is the SMA resistance in martensite finish temperature (relaxed SMA), $L_{R}(\Omega / m)$ is the linear resistance and $R_{A f}(\Omega)$ is the resistance at austenite finish temperature (i.e. at maximum contraction). 

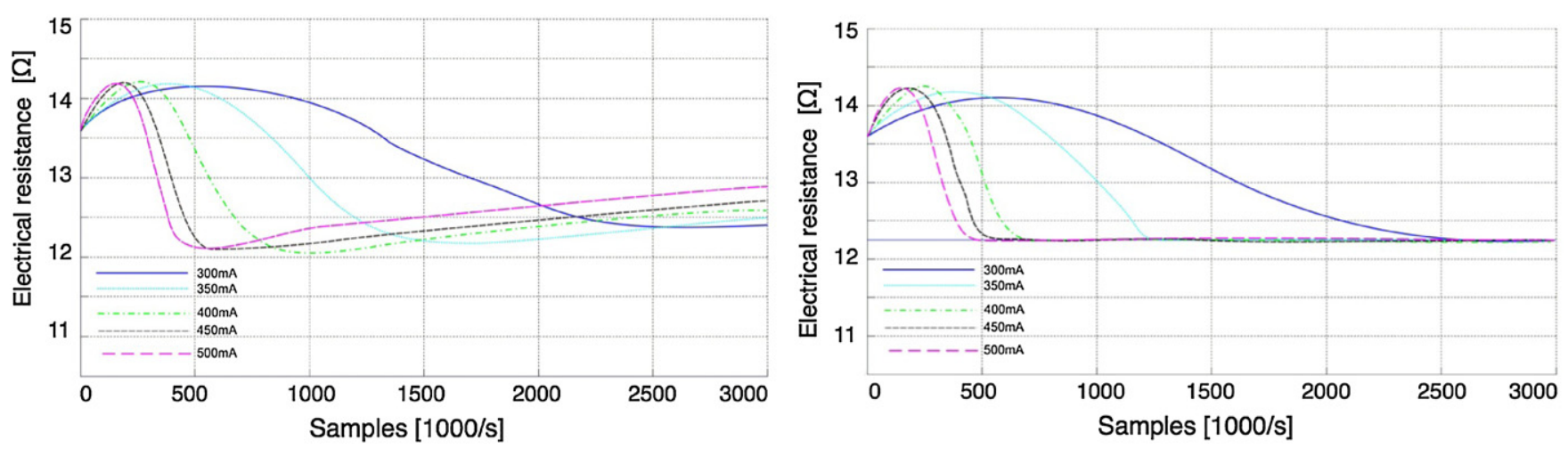

Figure 11. Experimental response of the SMA actuators for (left) open loop, and (right) closed loop with PID controller. A set of different current profiles ranging from 300 to $500 \mathrm{~mA}$ has been applied to the actuator. During open-loop behavior, the electrical resistance $(R)$ varies with time, it being difficult to maintain a desired bending angle position $(\beta)$ of the structure. By feeding back resistance measurements and closing the loop with the PID controller, the resistance curve is controlled to the desired set point $(12.25 \Omega)$. The plots have been postprocessed in order to improve readability.

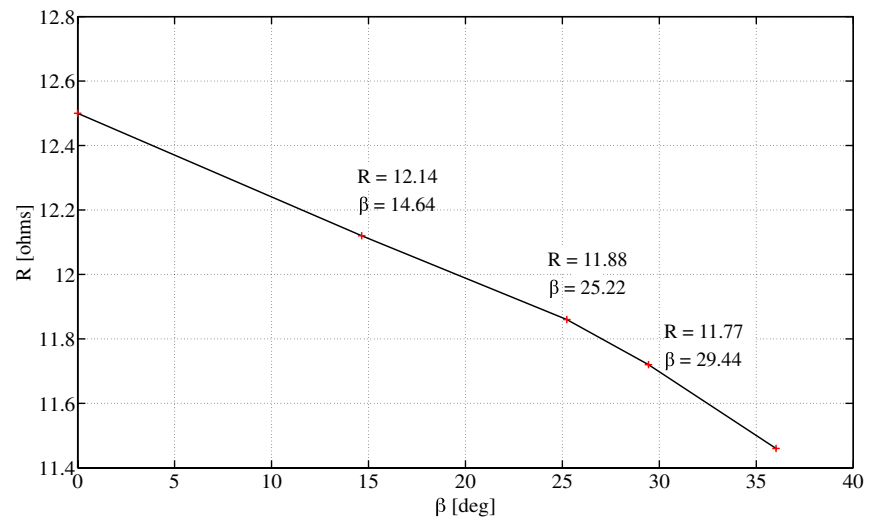

Figure 12. SMA electrical resistance measurements versus the bending angle $\beta$ of the mock-up.

Table 1. (Experimental) summary of the control performance in terms of SMA's resistance error, SMA contraction length error and structure bending angle error.

\begin{tabular}{llllll}
\hline $\begin{array}{l}\text { Target } \\
\text { resistance } \\
(\Omega)\end{array}$ & $\begin{array}{l}\text { Target } \\
\text { length } \\
(\mathrm{mm})\end{array}$ & $\begin{array}{l}\text { Bending } \\
\text { angle } \\
(\mathrm{deg})\end{array}$ & $\begin{array}{l}\text { Resistance } \\
\text { error } \\
(\Omega)\end{array}$ & $\begin{array}{l}\text { Length } \\
\text { error } \\
(\mathrm{mm})\end{array}$ & $\begin{array}{l}\text { Bend } \\
\text { error } \\
(\mathrm{deg})\end{array}$ \\
\hline 12.14 & 173.1 & 14.64 & $0.095(0.8 \%)$ & 1.385 & 5.99 \\
11.88 & 169.4 & 25.22 & $0.086(0.7 \%)$ & 1.186 & 2.81 \\
11.77 & 167.4 & 29.44 & $0.019(0.2 \%)$ & 0.335 & 0.69 \\
11.48 & 163.7 & 36 & $0.057(0.5 \%)$ & 0.819 & 1.36 \\
\hline
\end{tabular}

As can be noticed, the precision obtained is satisfactory, obtained as resistance variations at different input current profiles (see figure 12), with error percentages of less than $1 \%$ compared to the simulation (theoretical values). Only for one value (corresponding to the smallest strain at resistance of $12.14 \Omega$ ) was the precision obtained not satisfactory, since it induces a bending error of almost $6^{\circ}$. The error was expected to be smaller for higher temperatures, due to the hysteresis in the resistance, as also reported in [22]. For all the other values, from a practical point of view the precision obtained is sufficient as the bending error has a negligible impact on the mechanics of swimming.

\section{Swim patterns}

Once a suitable control for the actuators has been obtained, the next step is demonstrating that the mechatronic design is actually capable of reproducing standard swimming patterns. Figure 3 qualitatively illustrates the undulatory movement achieved by the body and tail segments. This section addresses this step quantitatively.

Swim patterns can be divided into two categories: periodic and aperiodic. Periodic swimming refers to cruise (steady) swimming and in-cruise turns, while aperiodic swimming refers to sudden changes of directions (also referred to in the literature as 'snap' turns) and fast starts.

\subsection{Steady swimming (cruise straight)}

Pioneer work on swimming patterns is due to [29, 37, 38]. For steady forward swimming, the body motion function can be described by the following equation:

$$
y=f_{S}(x, t)=\left(c_{1} x+c_{2} x^{2}\right) \sin \left(\frac{2 \pi}{\lambda} x+\omega t\right),
$$

where $x$ is the longitudinal position with respect to the head of the fish and $y$ is the lateral displacement. The $c_{1}$ and $c_{2}$ parameters define the wave amplitude, $\lambda$ is the wave length and $\omega$ is the wave frequency. The curve $f_{S}$ is transformed into the curve $f_{T}$, representing the position relative to the head [39]:

$$
y=f_{T}(x, t)=\left(c_{1} x+c_{2} x^{2}\right) \sin \left(\frac{2 \pi}{\lambda} x+\omega t\right)-c_{1} x \sin (\omega t) .
$$

For modeling purposes, the robot fish is implemented as a discrete number of elements, and the propagated wavefunction must be discretized (approximated by segments) in order to be reproduced. Therefore, the function that describes the wave propagation is defined as a sinusoidal-based time-dependent joint angle function $q_{j}$, where $j$ is the joint index [39]:

$q_{j}(t)=a_{j} \cdot \sin \left(\omega t+\phi_{j}\right), \quad j=1 \ldots$ number of joints. 


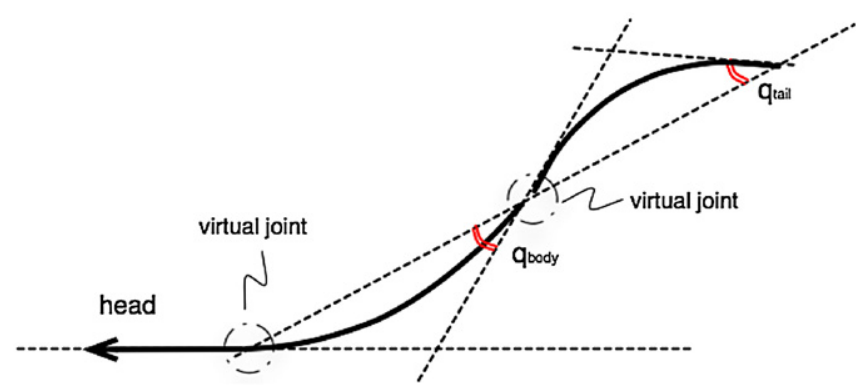

Figure 13. Virtual joints approximation. A joint position $q_{j}$ is equivalent to a bending angle $\beta_{j}=q_{j}$ w.r.t. the tangent of the previous segment's end $(j \in\{$ body, tail $\})$.

In our system, only the last two divisions are used for steady swimming ( $j \in\{$ body, tail $\}$; number of joints $=2$ ). In order to analyze and simulate the system, we consider that these two virtual joints are governed using (6), where bending $\beta_{j} \equiv q_{j}$ (see figure 13). Since the actuators bend the structure into arcs, the curve in (4) is approximated by circles (see figure 14).

\subsection{Cruise-in turning}

During steady swimming, smooth changes of direction, referred to as in-cruise turning, can be modeled as an asymmetry on the undulation with respect to the longitudinal axis. This can be modeled adding a bias function that defines a deflection curve:

$$
y=f_{S}(x, t)+d(x) .
$$

On the practical side, this implies that the joint equation in (6) becomes

$$
\beta_{j}(t)=q_{j}(t)=a_{j} \cdot \sin \left(\omega t+\phi_{j}\right)+b_{j},
$$

where the quantity $b_{j}$ is related to the curvature radius of the turn. For articulated bodies, it is easy to see that the bias $b_{j}$ for each joint and the direction $h$ of the last body w.r.t the first is $b=h / n, n$ being the number of joints. On the other hand, for a circular arc of length $L$, the relationship between its radius $r$ and the central angle $\theta$ is $r=L / \theta$. Since $h=\theta$, we have that

$$
b_{j}=\frac{L}{n \cdot r} \text {. }
$$

In our case, $L=0.3 \mathrm{~m}, n=2$ (virtual) joints, and the bias parameters $b_{j}$ in (9) can be easily calculated given the desired turning radius $r$.

\section{3. $C$-starts}

This kind of aperiodic pattern is used for fast turns in response to external stimuli (e.g. for escaping from a menace or for capturing prey). It comes in two ways: C-turns and S-turns, referring to the shape the fish takes during the maneuver. In real fish, such maneuvers take the order of milliseconds, and are activated by white (fast-twitch) muscles. It must be pointed out that our SMA actuator takes about $0.5 \mathrm{~s}$ (overloaded) to achieve the maximum curvature of $36^{\circ}$. This is why it makes sense to adopt them as slow-twitch (red) muscles for steady swimming. Nonetheless, in order to test the limits and possibilities of our concept regarding SMA technology,

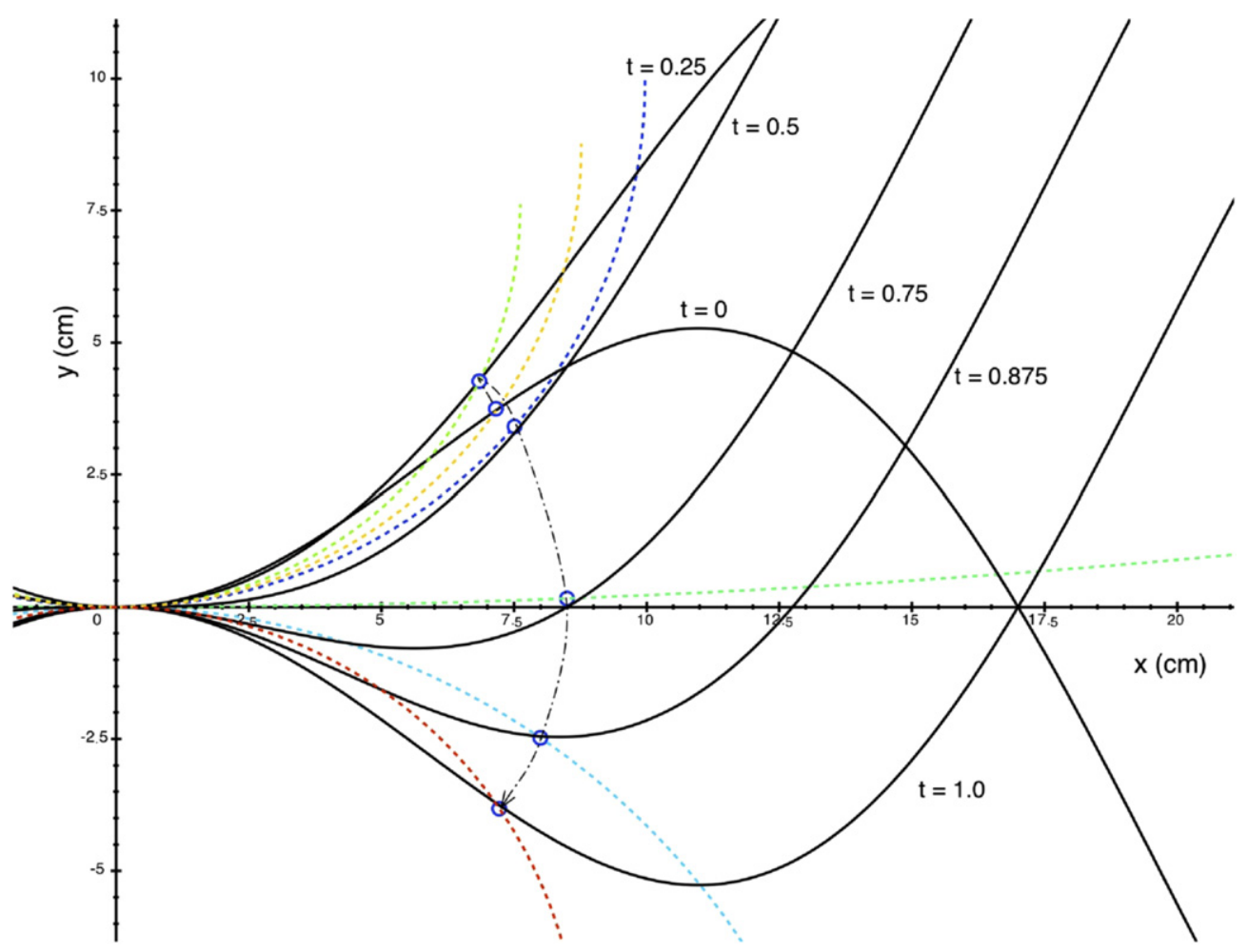

Figure 14. Approximation of $f_{T}(x, t)$ (solid lines) with circle arcs (dotted lines). $\omega=-\pi, c_{1}=\frac{4.5479}{L}, c_{2}=0, \lambda=\frac{2 \pi}{4 L}$. The blue circles represent the end point position of the fish bone segment of $L=8.5 \mathrm{~cm} . c_{1}$ corresponds to the maximum achievable bending and $\lambda$ has been set for subcarangiform swimming, where half a wave length is reproduced by the body consisting of two segments. The trajectory of the end point of the fishbone segment is shown by the arrow. 


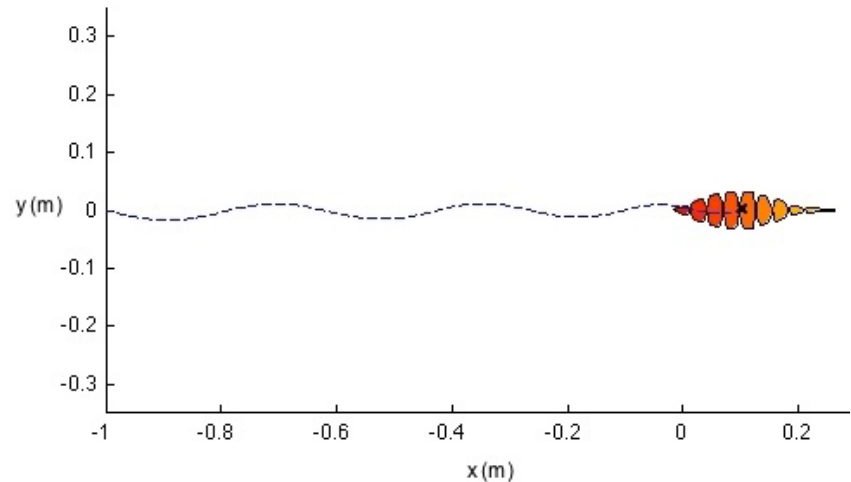

(a)

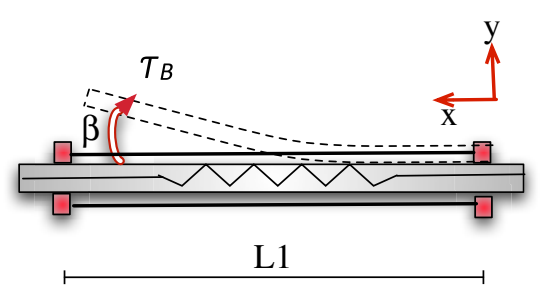

(c)

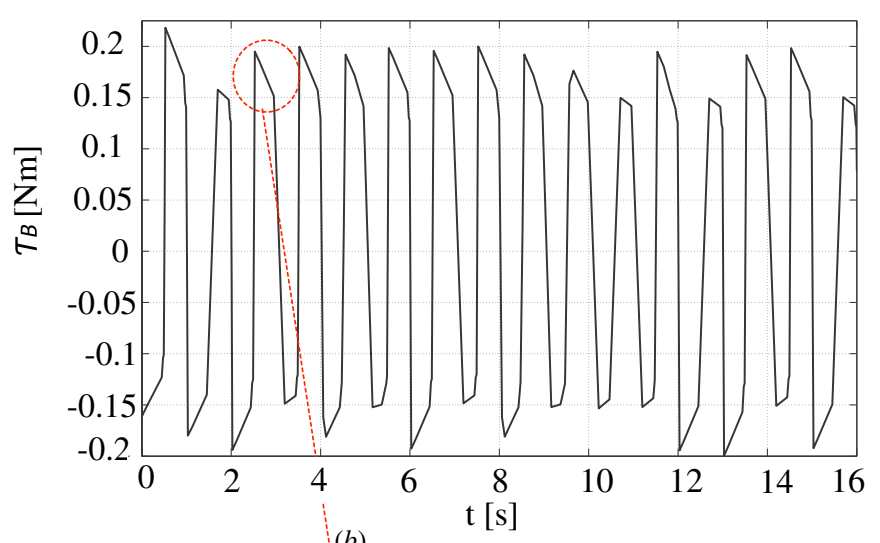

(b)

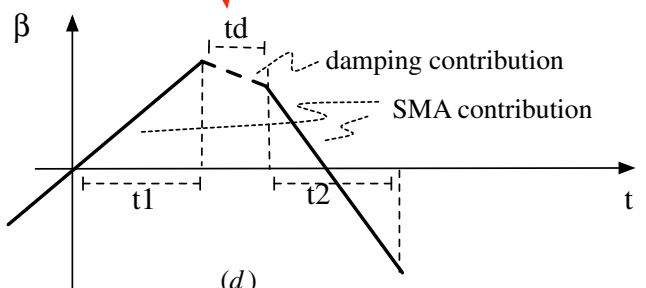

(d)

Figure 15. (a) Steady forward swimming $\left(a_{\text {tail }}=0.54, a_{\text {body }}=a_{\text {tail }} / 2, \phi_{\text {tail }}=-\pi / 4, \phi_{\text {body }}=0\right.$, tail-beat frequency $=1 / 2$ Hz). $(b)$ Simulation of the torsional torque $\tau_{\beta}$ required to bend the polycarbonate structure by using the V-shaped SMA actuators at $t_{c}=0.5 \mathrm{~s}$. $(c)$ Top view of the antagonistic V-shaped wires fixed to the backbone. For modeling, the bending property of the backbone is considered as a spring. $(d)$ Bending angle $\beta$ profile during SMA contraction: during $t_{1}$, the active actuator contracts upon heating, achieving a bending angle of $36^{\circ}$, subsequently, during $t_{d}=200 \mathrm{~ms}$, both pair of antagonistic actuators remain passive, and the decrease of the bending angle is provided by the restoring force caused by the polycarbonate structure trying to recover its original shape (i.e. spring-damping force). During $t_{2}$ the antagonistic actuator turns active providing the opposite motion.

we have implemented and simulated one such 'fast' start, the C-shaped.

\section{Simulations}

In order to assess the effectiveness of the proposed structure, we have conducted extensive numerical simulations, using the Bio-hydrodynamics Toolbox [40] for Matlab ${ }^{(\mathrm{TM})}(\mathrm{BhT})$. Such a toolbox provides a simple but thorough simulation tool. It allows us to perform numerical simulation involving 2D motions of rigid bodies in an ideal fluid. BhT is based on the Lagrangian formalism (least action principle).

The toolbox requires the model to be composed of articulated bodies. Shape changes between the bodies generate hydrodynamic forces and torques by which the bodies propel and steer themselves. Such a physical system based on both solid mechanics and fluid mechanics is called a fluidstructure interaction system. The fluid model of BhT is for a perfect fluid, and water pressure does not cause the body to bend.

For the purpose of the simulation, the body of the fish was discretized into nine parts (three for each fish body segment), with a mass proportional to the corresponding section of the fish body. The density of the material was set equal to the density of the water (neutral buoyancy), whereas the model's weight is 200 grams.
In order to model the torsional torque $\tau_{\beta}$ required to bend the polycarbonate backbone's structure and thus achieve the angle $\beta$, the V-shaped SMA actuators and the polycarnobate structure have been modeled as a springlike mechanism that provides a restoring force $F=-k X$ after bending. The torsional torque is described as $\tau_{B}=$ $J_{i} \ddot{\beta}+c \dot{\beta}+F_{\text {pull, max }} \cos \beta-k h L_{1}$, being the structure's moment of inertia $J_{i}=2.21 \times 10^{-5} \mathrm{~kg} \mathrm{~m}^{2}$, and the bending angular rate and acceleration $\dot{\beta}, \ddot{\beta}$, respectively. The term $c$ is the torsional damping coefficient obtained from the average values of computed strain forces of the SMAs (see the phenomenological model in the appendix), $k=0.2987 \mathrm{~N} \mathrm{~m}^{-1}$ corresponding to the spring constant property of the structure when recovering its shape after bending, $h=0.01 \mathrm{~m}$ is the maximum displacement of the structure when bending and $L_{1}$ is the length of the polycarbonate segment. The term $F_{\text {pull,max }}$ corresponds to the theoretical maximum pull force of the SMA wire, about $230 g-F$. Figure $15(c)$ shows the model, and figures $15(b)$ and $(d)$ show a plot of the required bending torque.

\subsection{Steady swimming}

Given the kinematic characteristics of our system, and based on the observation of fish swimming, the quantities described in (6) correspond to $a_{\text {body }}=a_{\text {tail }} / 2, \phi_{\text {tail }}=-\pi / 4, \phi_{\text {body }}=0$. $a_{\text {tail }}=0.49$ for nominal SMA contraction (4\%), and $a_{\text {tail }}=$ 0.54 for overloaded SMA contraction $(6 \%)$. 
Table 2. Summary of the performances of the simulations on steady swimming.

\begin{tabular}{llll}
\hline $\begin{array}{l}\text { Maximum } \\
\text { amplitude }\left(a_{\text {tail }}\right)\end{array}$ & $\begin{array}{l}\text { Tailbeat } \\
\text { frequency }(\mathrm{Hz})\end{array}$ & $\begin{array}{l}V \\
\left(\mathrm{~m} \mathrm{~s}^{-1}\right)\end{array}$ & $\begin{array}{l}V_{B L} \\
(\mathrm{~Hz})\end{array}$ \\
\hline Half (0.25) & $1 / 8$ & 0.025 & 0.083 \\
& $1 / 4$ & 0.025 & 0.083 \\
Nominal (0.49) & $1 / 2$ & 0.025 & 0.083 \\
& $1 / 8$ & 0.046 & 0.15 \\
Overloaded (0.54) & $1 / 4$ & 0.048 & 0.16 \\
& $1 / 2$ & 0.052 & 0.17 \\
& $1 / 2$ & 0.093 & 0.31 \\
& & 0.105 & 0.35 \\
& & 0.114 & 0.38 \\
\hline
\end{tabular}

Figure 15(a) shows an example of the trajectory followed by the fish. Table 2 reports the simulation results for steady swimming for various combinations of the maximum amplitude and frequency of the undulation (linear speed). The best values for the linear speed $V$ are achieved for the largest amplitudes, which can be obtained overloading the SMA as described earlier. As expected, such currents induce a further stress on the SMAs, but it is worth noting that, due to the oscillatory nature of the actuation, high peaks are maintained only for short periods of time. On the other hand, for low amplitudes, the speed achieved has no significant differences as far as tail-beat frequency is concerned.

\subsection{Morphology parameters}

Besides the linear speed $V$, some other parameters are useful for evaluating quantitatively the soundness of the design. Speed is expressed as body lengths $(L) \mathrm{s}^{-1}, V_{B L}=V / L$, and results are reported in the last column of table 2 .

The head swing factor $S_{h}$ is the ratio between the head oscillation amplitude induced by body motion and the tail tip oscillation amplitude $\left(S_{h}=A_{\text {head }} / A_{\text {tail }}\right)$. It ranges between 0.15 and 0.4 in real fish. High values indicate that a large amount of energy is wasted because the head oscillates too much and has to push water to the sides. The best result achieved has been $S_{h}=0.19$, using the parameter configuration: $a_{\text {tail }}=0.49, a_{\text {body }}=0.27, f=1 / 2 \mathrm{~Hz}$.

Finally, the Strouhal number $S_{t}=f_{\text {tail }} \cdot A_{\text {tail }} / V$, where $f_{\text {tail }}$ is the tail-beat frequency, $A_{\text {tail }}$ is the tail-beat peak-peak amplitude and $V$ is the linear speed, refers to the wake vortexes generated by the fish tail. Its value for real fish lays in the range $0.25-0.35$. In the simulations, our robot fish achieved values in the range [0.12-0.73], depending on tail beat frequency and amplitude. The value corresponding to figure 15 is $S_{t}=0.41$.

\subsection{In-cruise turning}

Figure 16 shows the result for the in-cruise turning maneuver. The turning radius resulting from the simulations is in good agreement with the theoretical values calculated in (9). Using the same relationship, we can compute the minimum turning radius of the robot. Given that the maximum biases $b_{\text {tail }}$ and $b_{\text {body }}$ are equal to the maximum amplitudes $a_{\text {tail }}, a_{\text {body }}$,

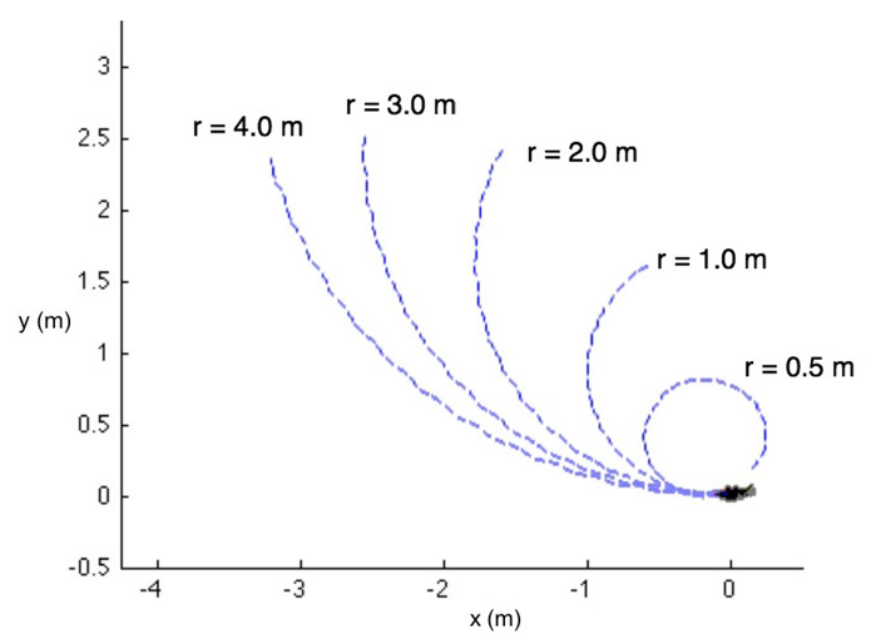

Figure 16. In-cruise turning. Labels refer to the desired turning radius (meters), corresponding (from left to right) to $b_{j}=0.0375,0.05,0.075,0.15,0.3$ (see (9)).

i.e. the oscillation is all at one side (e.g., 0 to $a_{\text {tail }}$ instead of $-a_{\text {tail }}$ to $\left.a_{\text {tail }}\right)$, the minimum radius will be $r_{\min }=\frac{L}{n \cdot b_{\max }}$. This corresponds to a minimum theoretical turning radius of $0.83 \mathrm{~m}$. In the simulations, the fastest turn (minimum turning radius $r$ ) has been achieved for the case of $r=0.5 \mathrm{~m}$, which corresponds to a maximum angular speed of $6 \mathrm{deg} \mathrm{s}^{-1}$.

\section{4. $C$-starts}

In this maneuver, all three segments of the fish are bent; the head also takes part in the maneuver. A third virtual joint located on the fish 'neck' (right behind the head) also activates. A sharp turning C-start maneuver implies contracting the muscles on the same side in a synchronized way (i.e. $\phi_{\text {tail }}=\phi_{\text {body }}=\phi_{\text {head }}$ ). The velocity and amplitude of the contraction induce the turning angle. Figure 20 (left) shows various stages of the best performing turn that can be obtained with a $1 \mathrm{~s}$ stroke of $28^{\circ}$, corresponding to nominal SMA current (300 mA current pulse, for a contraction time of $1 \mathrm{~s})$ for the three segments.

From the figure, it can be appreciated that the fish turns about $45^{\circ}$ in $1 \mathrm{~s}$ during the start stroke. After the starting stroke, the fish recovers the straight shape and begins steady swimming. During this period of time (approximately two tail beats) the fish holds the angular momentum generated with the initial stroke, and turns a further $15^{\circ}$, escaping with a final angle of approximately $60^{\circ}$.

\section{Experimental results}

We have tested the prototype in water in order to compare the real behavior with respect to the theory and the simulations.

The experimental setting is composed of a pool of $1 \times 0.5 \mathrm{~m}^{2}$, with a depth of about $70 \mathrm{~cm}$ and a grid of $5 \mathrm{~cm}$ resolution on the bottom. High-level swim patterns were generated using an off-board laptop computer and were programmed in Matlab ${ }^{(\mathrm{TM})}$. Reference positions (set points for angle $\beta$ ) were sent to the low-level control electronics via 
Table 3. Comparison of the simulation and experimental results for steady swimming (performance and morphology parameters).

\begin{tabular}{|c|c|c|c|c|c|c|c|c|}
\hline & \multicolumn{4}{|c|}{$f=0.5 \mathrm{~Hz}, a_{\text {tail }}=0.49$} & \multicolumn{4}{|c|}{$f=0.5 \mathrm{~Hz}, a_{\mathrm{tail}}=0.56$} \\
\hline & $\overline{V\left(\mathrm{~m} \mathrm{~s}^{-1}\right)}$ & $\mathrm{V}\left(\mathrm{BL} \mathrm{s}^{-1}\right)$ & $S_{t}$ & $S_{h}$ & $\mathrm{~V}\left(\mathrm{~m} \mathrm{~s}^{-1}\right)$ & $\mathrm{V}\left(\mathrm{BL} \mathrm{s}^{-1}\right)$ & $S_{t}$ & $S_{h}$ \\
\hline Simulation & 0.052 & 0.17 & 0.73 & 0.19 & 0.114 & 0.38 & 0.41 & 0.19 \\
\hline Real & 0.024 & 0.08 & 1.67 & 0.38 & 0.03 & 0.1 & 1.32 & 0.38 \\
\hline
\end{tabular}
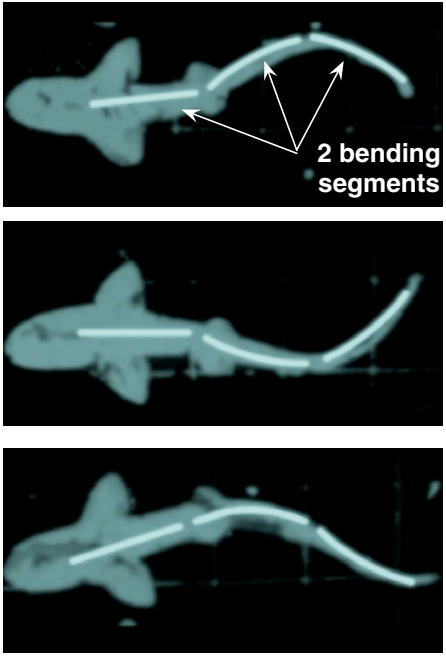

(a)
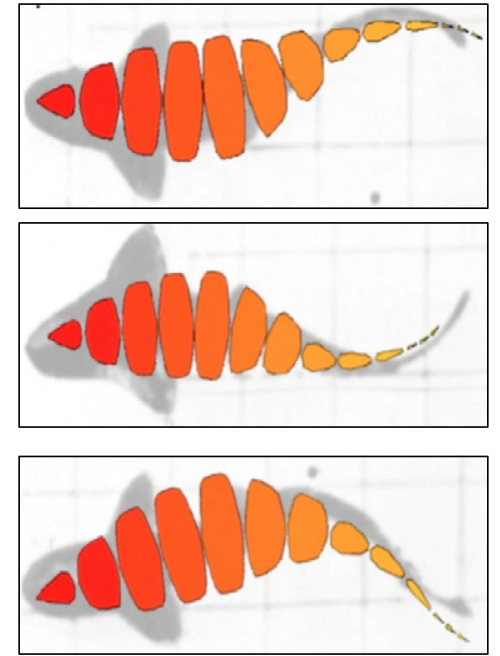

(b)

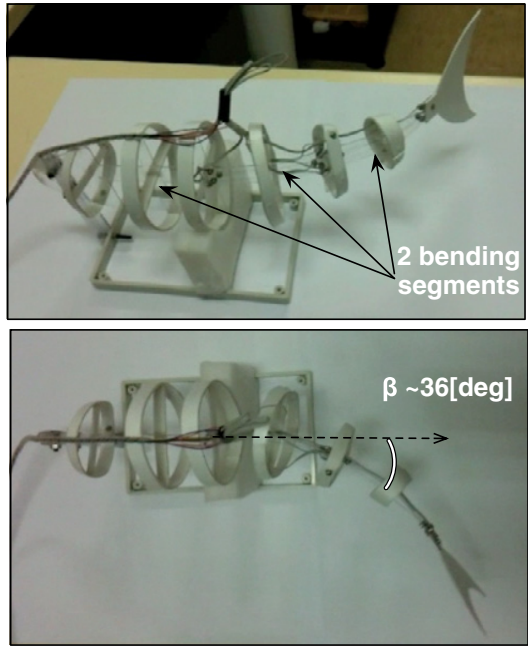

(c)

Figure 17. Comparison of qualitative assessment, numerical simulations and experimental results

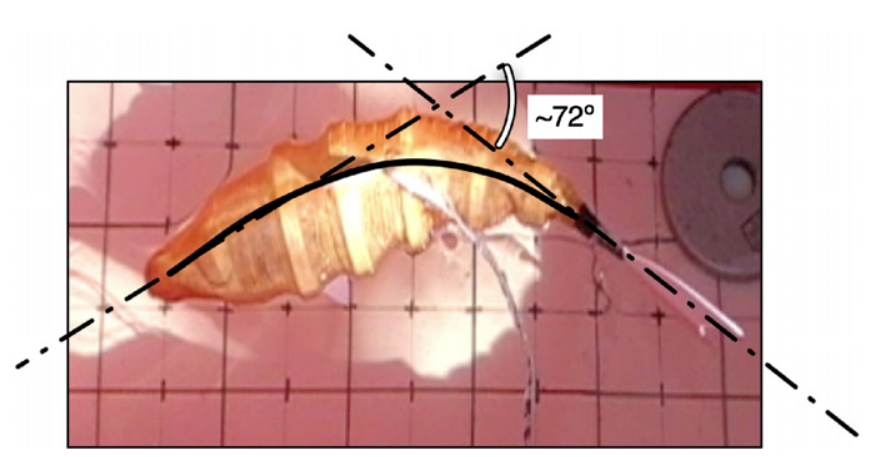

Figure 18. Testing bending in water (two segments, overloaded SMAs, open loop).

a USB interface. This produced the control signals for the actuators, sent through the tether using a standard I2C bus interface. Control electronics implemented closed-loop SMA control as depicted in figure 10.

First, the prototype was tested out of the water to measure actual bending angles. Figure 17 shows how actual bending is in good accordance with the theoretical angles.

Second, we qualitatively tested the effect of water pressure on the bending movement in open loop. Actuation speed and strain were not significantly affected, demonstrating that the actuators actually produced enough force to push the water aside (see figure 18).

Then, steady forward swimming tests were performed. The resulting linear speed was measured in order to calculate the performance parameters described above and reported in table 3. In summary, the experimental test has shown a significant difference from the simulation results. Differences were expected, since the model used for numerical simulation was simplified: as mentioned, it was a $2 \mathrm{D}$ model moving in a perfect fluid (i.e. no vorticity was considered), and the body was discretized into nine ellipse-shaped bodies. However, a dramatic degradation of the closed-loop swimming performances was observed. An explanation of this is mainly due to the protective skin, that turned out to be not elastic enough, and by the control tether, whose rigidity hindered the fish movements. The skin caused the actual bending range to suffer a reduction of almost 50\% (see figure 19) producing a very slow linear speed, approximately one fourth to one half of that obtained in the simulations. Moreover, the tether caused an unmeasurable lateral and longitudinal drag not considered in the simulations, and that introduced a noise in the free swimming, with a negative impact on the linear speed.

Finally, the C-start maneuver was performed. In this case, since the control produced a single strong pulse, the behavior was similar to the open loop test, and performances were much better, in good accordance with the simulation. Figure 20 shows a comparison, where only a small delay of about $0.5 \mathrm{~s}$ can be noticed.

\section{Final remarks and conclusions}

We have presented our current work on bio-inspired locomotion for underwater robots. The objective of this work is to investigate new kinds of robots capable of changing their 

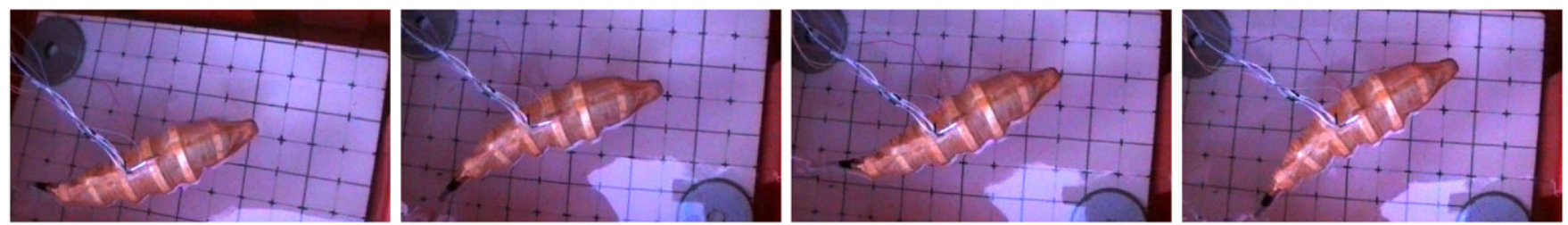

Figure 19. Linear swimming with $\mathrm{f}=0.5 \mathrm{~Hz}, a_{\text {tail }}=0.49$ at $t=1, t=2, t=3, t=4$ seconds (two tail beats). The distance travelled is approximately $7 \mathrm{~cm}$. Notice the reduction of the bending with respect to figure 18 .
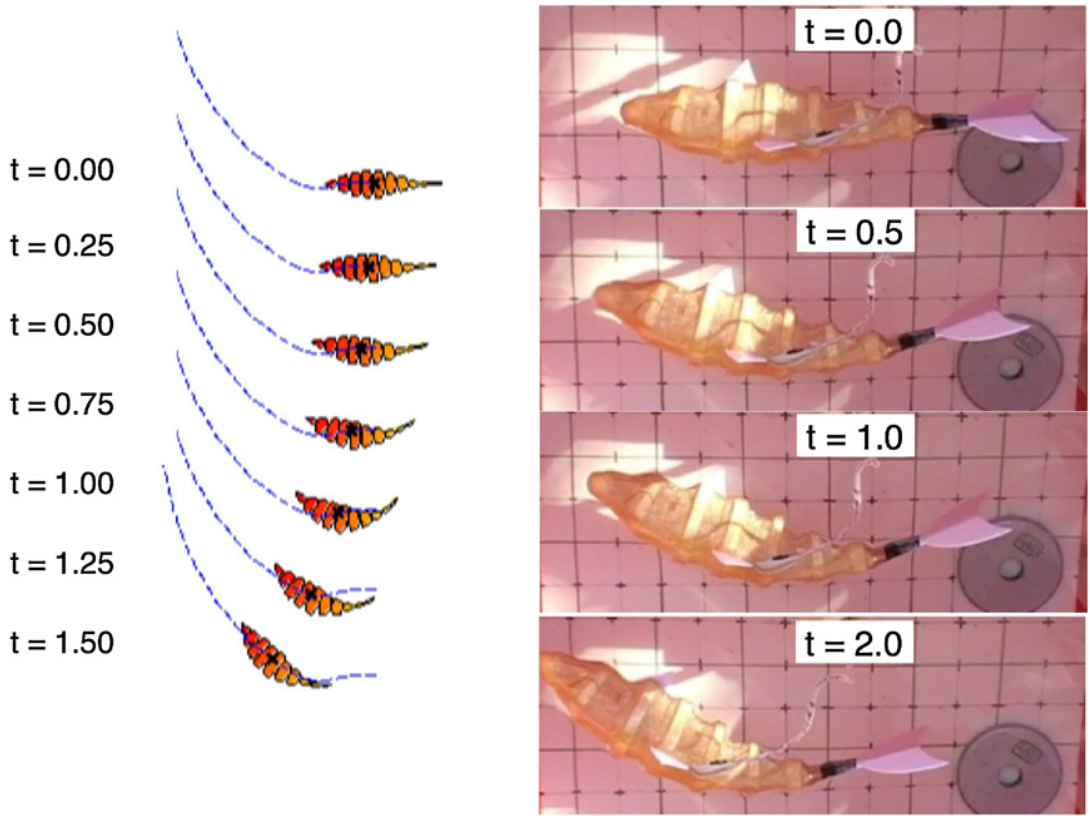

Figure 20. Stills of the C-start maneuver of the simulation and with the real prototype.

shape in a continuous way, and to investigate the use of active materials such as (but not limited to) SMAs as alternative actuation systems for such kind of robots.

The results of the modeling and of the numerical simulations confirm that the concept allows the robot to perform the main maneuvers according to the theory and models of fish swimming.

We would like to point out that the aim of our work is not to build a 'better' robot fish, but rather to experiment and elaborate on the concept of gear-less and motor-less robots, in order to develop the understanding of a technology that may be applied to the next generation of robots. In fact, compared to 'classic' robotic theory, mathematical models, simulation tools, control techniques and general understanding regarding such a concept is still in the embryonic stage, and work has to be done before it can be competitive with classical mechatronic designs.

In terms of the mechatronics design, the simplicity, extremely light weight and practically null volume of the actuators (an advantage of the available payload) make the fishlike robot suitable for biological applications. The actuators are absolutely silent and do not produce any vibration, a feature which can be exploited, e.g., in the observation of sea wild life since the robot would not disturb in any way (besides its presence). Beyond that, the novelty of using a bendable structure as the backbone of the fish based on the
V-shape configuration of antagonistic SMA-based actuation muscles has great potential for improving the maneuverability of the fish while performing the aforementioned swim patterns underwater.

In terms of control, the accuracy of the actuators has been improved in terms of avoiding the SMA fibers becoming slack due to the two-way shape memory effect of the antagonistic operation. The pre-heating approach demonstrated an increase in actuation speed, doubling the actuation frequency. The experimental results obtained have demonstrated the feasibility of the concept and, although not entirely satisfying, are very encouraging.

In conclusion, we believe that smart actuators and flexible continuous structures can be a promising field for making alternative bio-inspired robots, devoid of rotating parts and that are simpler and lighter, and that can have interesting application domains.

\section{Appendix. SMA thermo-mechanical model}

This appendix details further simulation results of SMA response based on thermo-mechanical behavior (phenomenological model from [33]) that relates the SMA strain $\varepsilon$, stress $\sigma$, austenite/martensite states $\xi$ and temperature $T$. Table A1 details the overall model, which is composed 

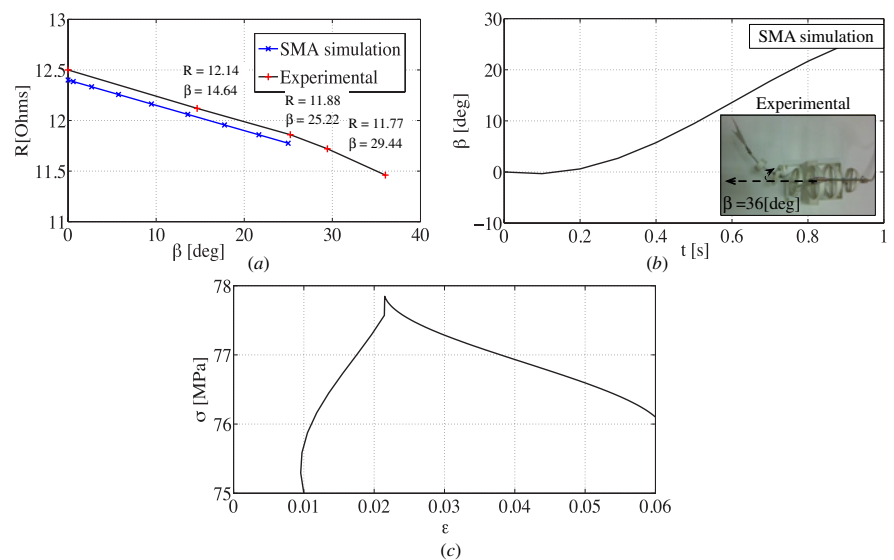

Figure 21. SMA simulation of thermo-mechanical behavior. $(a)$ linear variation between SMA strain and electrical resistance change $(R)$. The change of bending angle $\beta$ depends on the strain rate of the SMA. The slope of the linear function is likely similar in both experimental and simulated results (about $R=-0.0245 \beta+12.5$ ). (b) Bending angle obtained in simulation using an input current of $500 \mathrm{~mA}$, and actuation frequency of $f=1 \mathrm{~Hz}$. (c) Stress-strain relationship of one SMA actuator responding to an input current of $500 \mathrm{~mA}$.

Table A1. Thermo-mechanical model of SMA behavior.

\begin{tabular}{lllll}
\hline Variable & Model & Parameters & Description & Value (unit) \\
\hline Temperature & Heating: & $m_{s}, R_{s}, I_{s}$ & Mass, resistance, current & $1.14 \times 10^{-4}(\mathrm{~kg}), 12.5(\Omega)$ \\
$(T)$ & $m_{s} c_{p} \dot{T}=I_{s}^{2} R_{s}-h_{c} A_{c}\left(T-T_{o}\right)$ & $A_{c}$ & Wire area & $1.76 \times 10^{-8}\left(\mathrm{~m}^{2}\right)$ \\
& Cooling: & $h_{c}$ & Heat convection coefficient & $150\left(\mathrm{~J} \mathrm{~m}^{-2}{ }^{\circ} \mathrm{C}^{-1} \mathrm{~s}^{-1}\right)$ \\
& $m_{s} c_{p} \dot{T}=-h_{c} A_{c}\left(T-T_{o}\right)$ & $C_{p}$ & Specific heat & $0.2 \mathrm{kcal} \mathrm{kg}^{-1} \mathrm{C}^{-1}$ \\
Stress $(\sigma)$ & Heating: & $\Omega$ & Phase transformation & $-1.12(\mathrm{GPa})$ \\
& $\dot{\sigma}=\frac{\theta_{s}-\Omega\left(A_{f}-A_{s}\right)^{-1}}{1-\Omega\left(A_{f}-A_{s}\right)^{-1} C_{m}} \dot{T}$ & $\theta_{s}$ & Thermal expansion factor & $0.55\left(\mathrm{MPa}{ }^{\circ} \mathrm{C}^{-1}\right)$ \\
& Cooling: & $C_{m}, C_{a}$ & Stress effect & $10.3\left(\mathrm{MPa}{ }^{\circ} \mathrm{C}^{-1}\right)$ \\
& $\dot{\sigma}=\frac{\theta_{s}-\Omega\left(M_{s}-M_{f}\right)^{-1}}{1-\Omega\left(M_{s}-M_{f}\right)^{-1} C_{a}} \dot{T}$ & $A_{s}, A_{f}, M_{s}, M_{f}$ & Temperature: austenite, & $68,78,52,42\left({ }^{\circ} \mathrm{C}\right)$ \\
& & & martensite & \\
Strain $(\varepsilon)$ & Heating: & $E_{A}$ & & \\
& $\dot{\varepsilon}=\frac{\dot{\sigma}-\theta_{s} \dot{T}-\Omega \xi}{E_{A}}$ & $E_{M}$ & Austenite Young modulus & $75(\mathrm{GPa})$ \\
& Cooling: & & Martensite Young modulus & $28(\mathrm{GPa})$ \\
& $\dot{\varepsilon}=\frac{\dot{\sigma}-\theta_{s} \dot{T}-\Omega \xi}{E_{M}}$ & $\xi_{m}, \xi_{a}$ & FM constants & $1,0(\mathrm{dimensionless})$ \\
FM $(\xi)$ & Heating: & $a_{A}$ & Austenite amplitude factor & $0.31\left({ }^{\circ} \mathrm{C}^{-1}\right)$ \\
& $\xi=\frac{\xi_{m}}{2}\left[\cos \left(a_{A}\left(T-A_{s}\right)+b_{A} \sigma\right)+1\right]$ & $a_{M}$ & Martensite amplitude factor & $0.31\left({ }^{\circ} \mathrm{C}^{-1}\right)$
\end{tabular}

by three sub-models: (i) the heat transfer model $(T)$, (ii) thermo-mechanical model $(\varepsilon, \sigma)$ and (iii) phase transformation kinematics $(\xi)$. The heat transfer model is based on the heat transfer law for an SMA element, which has been widely used by many researchers for SMA modeling. It relates the heating current to the temperature based on Joule heating and free convection cooling. The thermomechanical model shows the relationship between stress and strain, whereas the phase transformation model allows us to analyze the hysteresis loop of the SMA.

Figure 21 shows the response of the SMA antagonistic arrangement. The PID controller feds each pair of SMAs with an average of maximum electrical current peaks of $550 \mathrm{~mA}$, which corresponds to the value of the overloaded behavior shown during the experimental results in figure 11. An important comparison between simulation and experimental data must be highlighted in terms of resistance change $(R)$ versus applied current $(I)$.

Note that in order to achieve a bending angle $(\beta)$ ranging from $0^{\circ}$ to about $36^{\circ}$, experimental measurements of electrical resistance variation decrease from 12.5 to $11.46 \Omega$ as a function of input current profile up to $0.5-0.6 \mathrm{~mA}$. Despite the phenomenological model reporting similar resistance variation compared to the experimental model, the maximum bending angle achieved at that current input is about $26^{\circ}$. This error appears since the $\mathrm{V}$-shaped mechanical arrangement model has not been taken into account within the thermo-mechanical equation that relates (i) pull-force caused by SMA strain and (ii) the bending angle achieved by the torsion torque applied to the polycarbonate structure. As a consequence, the simulated pull-force of the SMA wire is smaller, causing a smaller bending angle in contrast with the experiment. 


\section{References}

[1] Hu H 2006 Biologically inspired design of autonomous robotic fish at Essex IEEE SMC UK-RI Chapter Conference on Advances in Cybernetic Systems (Sheffield, 7-8 September) pp 3-8

[2] Anderson J M and Chhabra N K 2002 Maneuvering and stability performance of a robotic tuna Integr. Comp. Biol. 42 118-26

[3] Morgansen K A, Triplett B I and Klein D J 2007 Geometric methods for modeling and control of free-swimming fin-actuated underwater vehicles IEEE Trans. Robotics 23 1184-99

[4] Vela P A, Morgansenand K A and Burdick J W 2002 Underwater locomotion from oscillatory shape deformations IEEE Conf. on Decision and Control pp 2074-80

[5] Low K H and Chong C W 2010 Parametric study of the swimming performance of a fish robot propelled by a flexible caudal fin Bioinsp. Biomim. 5046002

[6] Lauder G V, Curet O M, Patankar N A and MacIver M A 2011 Mechanical properties of a bio-inspired robotic knifefish with an undulatory propulsor Bioinsp. Biomim. 6026004

[7] Valdivia P, Alvarado Y and Youcef-Toumi K 2006 Design of machines with compliant bodies for biomimetic locomotion in liquid environments ASME J. Dyn. Sys. Meas. Control $1283-13$

[8] Ming A, Park S, Nagata Y and Shimojo M 2009 Development of underwater robots using piezoelectric fiber composite IEEE Int. Conf. on Robotics and Automation pp 3821-6

[9] Nguyen Q S, Park H C and Byun D 2011 Thrust analysis of a fish robot actuated by piezoceramic composite actuators J. Bionic Eng. 8158164

[10] Wang Z, Hang G, Wang Y, Li J and Du W 2008 Embedded SMA wire actuated biomimetic fin: a module for biomimetic underwater propulsion Smart Mater. Struct. 17025039

[11] Zhang Y h, Song Y, Yang J and Low K H 2008 Numerical and experimental research on modular oscillating fin J. Bionic Eng. 5 13-23

[12] Wang Z, Wang Y, Jian L and Hang G 2009 A micro biomimetic manta ray robot fish actuated by SMA Proc. 2009 IEEE Int. Conf. on Robotics and Biomimetics (Guilin, February) pp 1809-13

[13] Zhang Z, Philen M and Neu W 2010 A biologically inspired artificial fish using flexible matrix composite actuators: analysis and experiment Smart Mater. Struct. 19094017

[14] Rediniotis O K, Wilson L N, Lagoudas D C and Khan M M 2002 Development of a shape-memory-alloy actuated biomimetic hydrofoil J. Intell. Mater. Syst. Struct. 13 35-49

[15] Zhang Y, Shangrong L, Ji M and Yang J 2005 Development of an underwater oscillatory propulsion system using shape memory alloy Proc. IEEE Int. Conf. on Mechatronics and Automation pp 1878-83

[16] Suleman A and Crawford C 2008 Design and testing of a biomimetic tuna using shape memory alloy induced propulsion Comput. Struct. 86491499

[17] Shatara S, Chen Z and Tan X 2010 Modeling of biomimetic robotic fish propelled by an ionic polymer-metal composite caudal fin IEEE/ASME Trans. Mech. 15 448-59

[18] Jung J, Kim B, Kim D and Park J 2005 A biomimetic undulatory tadpole robot using ionic polymermetal composite actuators Smart Mater. Struct. 14 1579-85

[19] Zio D, Tangorra J, Anquetil P, Fofonoff T, Chen A and Hunter I 2007 The application of conducting polymers to a biorobotic fin propulsor Bioinsp. Biomim. 2 S6-17
[20] Wang H, Tjahyono S S, Macdonald B, Kilmartin P A, Travas-Sejdic J and Kiefer R 2007 Robotic fish based on a polymer actuator Proc. Australasian Conf. on Robotics and Automation (Brisbane, December) pp 1809-13

[21] Teh Y H and Featherstone R 2008 An architecture for fast and accurate control of shape memory alloy actuators Int. J. Robot. Res. 27 595-611

[22] Meier H, Czechowicz A and Haberland C 2009 Control loops with detection of inner electrical resistance and fatigue-behaviour by activation of NiTi-shape memory alloys Eur. Symp. Martensitic Transformations (Prague, September) 05006 (doi:10.1051/esomat/200905006)

[23] Russell R A and Gorbet R B 1995 Improving the response of sma actuators IEEE Int. Conf. on Robotics and Automation (Nagoya, Japan) pp 2299-304

[24] Mohanchandra K P, Shin D D and Carman G P 2004 High frequency actuation of thin film NiTi Sensors Actuators A 111 166-71

[25] Ma C, Wang R, Sun Q, Zohar Y and Wong M 2002 Frequency response of TiNi shape memory alloy thin film micro-actuators 13th Int. Conf. on Micro Electro Mechanical Systems (MEMS 2000) (23-27 January) pp 370-4 (doi:10.1109/MEMSYS.2000.838545)

[26] Zohar Y, Wang R X and Wong M 2002 Residual stress-loaded titanium-nickel shape-memory alloy thin-film micro-actuators J. Micromech. Microeng. 12 323-7

[27] Sfakiotakis M, Lane D M and Davies J B C 1999 Review of fish swimming modes for aquatic locomotion IEEE J. Ocean. Eng. 24 235-52

[28] Ellerby D J, Altringham J D, Williams T and Block B A 2000 Slow muscle function of pacific bonito (sarda chiliensis) during steady swimming J. Exp. Biol. 203 2001-13

[29] Gray J 1933 Studies in animal locomotion J. Exp. Biol. 10 88-104

[30] Visintin A 1994 Differential Models of Hysteresis (Applied Mathematical Sciences vol 111) (Berlin: Springer)

[31] Kuribayashi K 1986 A new actuator of a joint mechanism using Ti-Ni alloy wire Int. J. Robot. Res. 4 47-58

[32] Tsukamoto M, Ikuta K and Hirose S 1991 Mathematical model and experimental verification of shape memory alloy for designing micro actuator Proc. IEEE Micro. Electro. Mech. Syst. (Nara, Japan, January-February) pp 103-8

[33] Tanaka K 1986 A thermomechanical sketch of shape memory effect: one-dimensional tensile behaviour Int. J. Struct. Mech. Mater. Sci. 18 251-63

[34] Tsukamoto M, Ikuta K and Hirose S 1988 Shape memory alloy servo actuator system with electric resistance feedback and application for active endoscope IEEE Int. Conf. on Robotics and Automation (Philadelphia, PA 24-29 April) pp 427-30

[35] Kuribayashi K 1991 Improvement of the response of an SMA actuator using a temperature sensor Int. J Robot. Res. $1013-20$

[36] Mihalcz I 2001 Fundamental characteristics and design method for nickel-titanium shape memory alloy Period. Polytech. Ser. Mech. Eng. $\mathbf{4 5}$ 75-86

[37] Lighthill M J 1960 Note on the swimming of slender fish J. Fluid Mech. 9 305-17

[38] Wu T Y T 1961 Swimming of a waving plate J. Fluid Mech. 10 321-44

[39] Liu J and Huosheng H 2007 Methodology of modelling fish-like swim patterns for robotic fish Proc. 2007 IEEE Int. Conf. on Mechatronics and Automation pp 1316-21

[40] Munnier A and Pincon B 2008 Biohydrodynamics toolbox http://bht.gforge.inria.fr/ 